\title{
The ALPINE-ALMA [CII] survey \\ Dust mass budget in the early Universe
}

F. Pozzi ${ }^{1,2}$, F. Calura ${ }^{2}$, Y. Fudamoto ${ }^{3}$, M. Dessauges-Zavadsky ${ }^{3}$, C. Gruppioni ${ }^{2}$, M. Talia ${ }^{1}$, G. Zamorani ${ }^{2}$, M. Bethermin ${ }^{4}$, A. Cimatti ${ }^{1,5}$, A. Enia ${ }^{1}$, Y. Khusanova ${ }^{4}$, R. Decarli ${ }^{2}$, O. Le Fèvre ${ }^{4}$, P. Capak ${ }^{6}$, P. Cassata ${ }^{7}$, A. L. Faisst ${ }^{6}$, L. Yan $^{8}$, D. Schaerer ${ }^{3}$, J. Silverman ${ }^{9}$, S. Bardelli ${ }^{2}$, M. Boquien ${ }^{10}$, A. Enia ${ }^{1}$, D. Narayanan ${ }^{11}$, M. Ginolfi ${ }^{12}$, N. P. Hathi ${ }^{13}$, G. C. Jones ${ }^{14,15}$, A. M. Koekemoer ${ }^{13}$, B. C. Lemaux ${ }^{16}$, F. Loiacono ${ }^{1}$, R. Maiolino ${ }^{14,15,17}$,

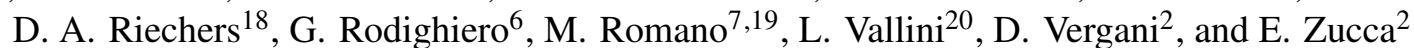

1 Dipartimento di Fisica e Astronomia, Universitá of Bologna, via Gobetti 93/2, 40129 Bologna, Italy e-mail: f.pozzi@unibo.it

2 INAF - Osservatorio di Astrofisica e Scienza dello Spazio di Bologna, via Gobetti 93/3, 40129 Bologna, Italy

3 Department of Astronomy, University of Geneva, 51 Ch. des Maillettes, 1290 Versoix, Switzerland

${ }^{4}$ Aix Marseille Univ. CNRS, LAM, Laboratoire d'Astrophysique de Marseille, Marseille, France

5 INAF - Osservatorio astrofisico di Arcetri, Largo E. Fermi 5, 50125 Firenze, Italy

${ }^{6}$ Infrared Processing and Analysis Center, California Institute of Technology, Pasadena, CA 91125, USA

7 Dipartimento di Fisica e Astronomia, Universitá di Padova, vicolo Osservatorio 3, 35122 Padova, Italy

8 The Caltech Optical Observatories, California Institute of Technology, Pasadena, CA 91125, USA

9 Kavli Institute for the Physics and Mathematics of the Universe, The University of Tokyo, 5-1-5 Kashiwanoha, Kashiwa-shi, Chiba 277-8583, Japan

10 Centro de Astronomia (CITEVA), Universidad de Antofagasta, Avenida Angamos 601, Antofagasta, Chile

${ }^{11}$ Department of Astronomy, University of Florida, 211 Bryant Space Sciences Center, Gainesville, FL 32611, USA

12 European Southern Observatory, Karl-Schwarzschild-Straße 2, 85748 Garching, Germany

13 Space Telescope Science Institute, 3700 San Martin Drive, Baltimore, MD 21218, USA

14 Cavendish Laboratory, University of Cambridge, 19 J. J. Thomson Ave., Cambridge CB3 0HE, UK

1516 Kavli Institute for Cosmology, University of Cambridge, Madingley Road, Cambridge CB3 OHA, UK

16 Department of Physics, University of California, Davis, One Shields Ave., Davis, CA 95616, USA

17 Department of Physics and Astronomy, University College London, London, UK

18 Department of Astronomy, Cornell University, Space Sciences Buildin, Ithaca, NY 14853, USA

19 INAF - Osservatorio Astronomico di Padova, Vicolo dell'Osservatorio 5, 35122 Padova, Italy

${ }^{20}$ Scuola Normale Superiore, Piazza dei Cavalieri 7, 56126 Pisa, Italy

Received 29 December 2020 / Accepted 25 May 2021

\section{ABSTRACT}

Aims. The dust content of normal galaxies and the dust mass density (DMD) at high- $z(z>4)$ are unconstrained given the source confusion and the sensitivity limitations of previous observations. The ALMA Large Program to INvestigate [CII] at Early times (ALPINE), which targeted 118 ultra-violet (UV)-selected star-forming galaxies at $4.4<z<5.9$, provides a new opportunity to tackle this issue for the first time with a statistically robust dataset.

Methods. We exploited the rest-frame far-infrared (FIR) fluxes of 23 galaxies individually detected in their continuum emission, as well as stacked continuum images, to measure the dust content of the $118 \mathrm{UV}$-selected ALPINE galaxies. We focused on the dust scaling relations and, by comparison with predictions from chemical evolution models, we probed the evolutionary stage of UVselected galaxies at high- $z$. By using the observed correlation between the UV luminosity and the dust mass, we estimated the DMD of UV-selected galaxies at $z \sim 5$, weighting the galaxies by means of the UV luminosity function. The derived DMD is compared with the value we estimated from ten ALPINE galaxies blindly detected in the FIR continuum, at the redshift of the ALPINE targets. Results. Our ALMA survey allows the exploration for the first time of the dust content in normal star-forming galaxies at $z>4$ in a statistically robust sample of sources. The comparison of the observed dust scaling relations with chemical evolution models suggests that ALPINE galaxies are not likely progenitors of disc galaxies, but of intermediate- and low-mass proto-spheroids, resulting in present-day bulges of spiral or elliptical galaxies. Interestingly, this conclusion is in line with the independent morphological analysis that shows that the majority $(\sim 70 \%)$ of the dust-continuum detected galaxies have a disturbed morphology. The DMD obtained at $z \sim 5$ from UV-selected sources is $\sim 30 \%$ of the value obtained from blind FIR-selected sources, showing that the UV selection misses the most dust-rich, UV-obscured galaxies.

Key words. dust, extinction - galaxies: ISM - galaxies: high-redshift

\footnotetext{
$\dagger$ Deceased.
} 


\section{Introduction}

Cosmic dust accounts for an almost negligible contribution to the baryon mass in the Universe $(\sim 0.1 \%$ in the local Universe; see Shull et al. 2012). Nevertheless, it plays a crucial role in many astrophysical and astrochemical aspects. In particular, it strongly affects the spectral energy distribution of galaxies, being a source of attenuation for UV-optical photons and an emission source in the infrared domain. Therefore, recovering the galaxy dust properties is of primary importance in order to achieve a selfconsistent understanding of the physics and evolution of galaxies across cosmic time.

Before the advent of the Atacama Large Millimeter Array (ALMA), dust emission of normal star-forming galaxies was detectable mainly thanks to the Herschel surveys up to $z<4(z \sim 2$, e.g., Rodighiero et al. 2011; Gruppioni et al. 2013; Magnelli et al. 2013; Lemaux et al. 2014) or extremely dusty (usually strongly lensed) galaxies up to high redshift ( $z>$ 4, e.g., Combes et al. 2012; Rowan-Robinson et al. 2016; Negrello et al. 2017).

Currently, ALMA is revolutionising this field of research thanks to its superb sensitivity and high spatial resolution; the problem of source confusion is thus avoided, allowing continuum detection at $z>4$ also for normal star-forming galaxies (i.e. with star formation rates from a few up to tens of solar masses/year).

Given its relatively small field of view $(\sim 30 \mathrm{arcsec}$ at $1.2 \mathrm{~mm}$ ), ALMA started the exploration of the high- $z$ Universe by targeting individual galaxies (e.g., Hodge et al. 2013; Riechers et al. 2014; Capak et al. 2015; Watson et al. 2015; Laporte et al. 2017). The number of normal to large programs over recent years has been steadily increasing, and different continuum surveys have been performed, ranging from the deepest observations over small areas $\left(<5 \operatorname{arcmin}^{2}\right.$, e.g., Dunlop et al. 2017; González-López et al. 2020) to the wider (a few tens of $\operatorname{arcmin}^{2}$ ) and shallower surveys (e.g., Franco et al. 2018).

Magnelli et al. (2020) carried out the first study of the dust mass density (DMD) from $z \sim 0.5$ up to $z \sim 5$, taking advantage of the deepest $\left(9.5 \mu \mathrm{Jy}_{\text {beam }^{-1}}\right)$ ALMA $1.2 \mathrm{~mm}$ continuum map of $\sim 4 \mathrm{arcmin}^{2}$ in the Hubble Ultra Deep Field (HUDF) from the ASPECS survey (Walter et al. 2016; Decarli et al. 2019; González-López et al. 2020). Their results confirm the presence of a peak around $z \sim 1-3$ and a decrease in the DMD from $z \sim 1$ down to the local Universe already found by Herschel (Dunne et al. 2011; Driver et al. 2018; Pozzi et al. 2020), the latter result being in contrast with cosmological simulations (e.g., Popping et al. 2017; Aoyama et al. 2018; Li et al. 2019).

In order to improve our understanding of the DMD at high redshift $(>4)$, in this work we take advantage of our recently completed ALMA Large Program to INvestigate [CII] at Early times (ALPINE, PI: Le Fèvre, see Le Fèvre et al. 2020; Béthermin et al. 2020; Faisst et al. 2020b). The goal of the ALPINE survey was to observe the prominent [CII] $158 \mu \mathrm{m}$ emission line for $118 \mathrm{UV}$-selected normal star-forming galaxies at $z \sim 4.4-5.8$. From 118 targets, 75 galaxies were detected in [CII] and 23 in continuum. In addition to the main targets, a blind search for continuum and line emitters in an $\sim 25 \operatorname{arcmin}^{2}$ area was also performed in the ALPINE pointings (Béthermin et al. 2020; Loiacono et al. 2021).

Over the last year many results based on the ALPINE survey have been presented, describing the interstellar medium (ISM) properties of normal star-forming galaxies at $z \sim 5$ (the [CII]-SFR relation: Schaerer et al. 2020; the [CII] outflows: Ginolfi et al. 2020b; the [CII] spatial scales: Fujimoto et al. 2020b; the Ly $\alpha$-[CII] velocity offset: Cassata et al. 2020; the IRX- $\beta$ relation: Fudamoto et al. 2020a; the gas content: Dessauges-Zavadsky et al. 2020, detailed studies on individual sources (Jones et al. 2020; Romano et al. 2020; Ginolfi et al. 2020a) or presenting statistical studies at $z \sim 5$, including the [CII] luminosity function for ultraviolet (UV) and serendipitously detected line emitters (Yan et al. 2020; Loiacono et al. 2021), the infrared (IR) luminosity function (Gruppioni et al. 2020), and the cosmic star formation rate density (SFRD, Khusanova et al. 2021).

In the present work we measure the dust content of UVselected normal (main-sequence) galaxies and IR continuum serendipitously detected sources. In Sects. 2 and 3 respectively the ALPINE sample and the measurements of the dust masses are presented. In Sect. 4 the derived ISM properties of the ALPINE targets (dust masses from the present work and gas masses from Dessauges-Zavadsky et al. 2020) are combined with the well characterised stellar masses and SFRs (Faisst et al. 2020 b) to discuss the dust scaling relation for the UV population at $z \sim 5$. In Sect. 5 the DMD for the UV-selected and for the IRselected populations are presented. Finally, in Sect. 6 we present our conclusions.

Throughout the paper, we assume a $\Lambda \mathrm{CDM}$ cosmology with $\Omega_{m}=0.3, \Omega_{\lambda}=0.7$, and $H_{0}=70 \mathrm{~km} \mathrm{~s}^{-1} \mathrm{Mpc}^{-3}$.

\section{Observations}

In the following section we describe in detail the ALPINE continuum detected sample that we use in our study. For a more complete description of the overall survey, the data reduction, and the ancillary data we refer to Le Fèvre et al. (2020), Béthermin et al. (2020), and Faisst et al. (2020b), respectively.

The 118 galaxies from the ALPINE survey (Le Fèvre et al. 2020) are rest-frame UV-selected galaxies at redshift $z \sim$ 4.4-5.9. These galaxies originated in two fields, namely the COSMOS Evolution Survey field (COSMOS, Scoville et al. 2007) and the Extended Chandra Deep Field South (ECDFS, Giacconi et al. 2002). They all have spectroscopic redshifts from different campaigns (see Faisst et al. 2020b for details). They were selected to be representative of star-forming main-sequence galaxies at $z \sim 5$ (i.e. Speagle et al. 2014). They have stellar masses in the range $M_{\star}=10^{8.4}-10^{11} M_{\odot}$ and star formation rates (SFRs) in the range SFR $=3-270 M_{\odot} \mathrm{yr}^{-1}$. The stellar masses are derived from spectral energy distribution (SED) fitting using broadband and medium-band (intermediate-band) rest-frame UV-optical photometry (see Faisst et al. 2020b for details). The SFRs reported are derived from Schaerer et al. (2020), using the ALPINE far-infrared (FIR) data, to properly account for dust-obscured SFRs. They are computed as SFR(UV)+SFR(IR) in the case of IR continuum detection, and are derived in the other cases from the observed UV slope and luminosity using the ALPINE IRX-beta relation (obtained from stacking, see Fudamoto et al. 2020a). These SFRs are globally consistent with the SFR derived from UV-optical SED-fitting (median (SFR(UV+IR)/SFR(SED)) 1.2). Since there are only a few outliers for which $\mathrm{SFR}(\mathrm{UV}+\mathrm{IR})$ results in a factor up to $\sim 5$ higher than SFR(SED), we prefer to consider the SFR(UV+IR) in the present analysis to properly account for the dust-obscured SFR.

The ALPINE targets were observed during Cycle 5 and Cycle 6 in Band $7(275-373 \mathrm{GHz})$, which covers the [CII] line from $z=4.1-5.9$. To avoid atmospheric absorption, no targets were included in the redshift range $z=4.6-5.1$. Each target was observed between 15 and $45 \mathrm{~min}$. The data 
were reduced and calibrated with standard routines using the Common Astronomy Software Application (CASA) software (McMullin et al. 2007). We refer to Béthermin et al. (2020) for all the details of the data reduction processes and the catalogue construction. The continuum maps, of interest for this work, were produced using line-free channels, reaching an angular resolution between $0.7^{\prime \prime}$ and $1.6^{\prime \prime}$ and mean sensitivities (RMS) of $50 \mu \mathrm{Jybeam}^{-1}$ and $28 \mu \mathrm{Jybeam}^{-1}$ in the $4.3<z<4.6$ and $5.1<z<5.9$ ranges, respectively. Some sources were marginally resolved at the angular resolution reached by the observations, and different methods were implemented and compared to measure the fluxes. The simulations performed (see Sects. 3.2 and 3.9 in Béthermin et al. 2020) show that the $2 \mathrm{D}$ fit photometry is the most accurate, even though other methods are consistent within the error bars. The final continuum catalogue consists of 23 sources detected at signal-to-noise ratio $S / N>3.5$ (see Table B.1 in Béthermin et al. 2020). In the case of multi-component objects (DEIMOS_COSMOS_881725, vuds_cosmos_5101209780 and vuds_efdcs_530029038) we used the sum of the components for DEIMOS_COSMOS_881725, while we used the fluxes of the central targets for vuds_cosmos_5101209780 and vuds_efdcs_530029038 since the companions are likely separated objects (see Appendix D.2 and Table D.1 in Béthermin et al. 2020 and Ginolfi et al. 2020a).

We note here that in Sect. 5.1 we perform a stacking analysis on the rest-frame $157 \mu \mathrm{m}$ ALMA continuum images, including both the 23 individual continuum detected sources and the nondetections. This is essential for recovering the average properties of the ALPINE population.

In the ALPINE survey, 57 sources were also serendipitously detected at 860-1000 $\mu \mathrm{m}$ (ALMA band-7), and we refer to Béthermin et al. (2020) for the description of the detection procedure and the delivery of the catalogue of non-targets. In this case, given the absence of a prior, a conservative threshold of $S / N>5$ was applied. We refer to Gruppioni et al. (2020) for the detailed characterisation of the sources, done by searching for counterparts of the serendipitous targets in all the available multi-band and photo- $z$ catalogues, and also performing new photometry. The resulting blind sources are distributed over a wide photometric redshift range (0.5-6) computed including UV, optical, and IR data; it was not possible to assign a redshift for only four sources (see Sect. 3.2.2 in Gruppioni et al. 2020). For the aims of the present work (see Sect. 5.3), we consider the subsample of ten galaxies in the redshift range corresponding to the ALPINE targets $(4.1<z<5.9)$. All ten sources are detected in the COSMOS field.

\section{Dust mass estimates}

We derive the dust masses using a single modified black body (MBB) curve, under the approximation of an optically thin regime (see Bianchi 2013),

$M_{\text {dust }}=\frac{D_{L}^{2} S_{v_{\text {obs }}}}{(1+z) k_{v} B_{v}(T)}$,

where $v$ and $v_{\mathrm{obs}}$ are the rest-frame and observed frequencies $\left(v=v_{\text {obs }}(1+z)\right), B_{v}(T)$ is the Planck function, $D_{L}$ the luminosity distance, $k_{v}$ the grain absorption cross section per unit mass, and $S_{v_{\text {obs }}}$ is the observed flux corresponding to a rest-frame frequency at which the dust can be considered optically thin.

We assume a power law for $k_{v}, k_{v}=k_{v_{0}}\left(\frac{v}{v_{0}}\right)^{\beta} \mathrm{cm}^{2} \mathrm{~g}^{-1}$. We adopt $k_{v_{0}}=4 \mathrm{~cm}^{2} \mathrm{~g}^{-1}$ with $v_{0}=1.2 \mathrm{THz}$ (see Bianchi 2013) and $\beta=1.8$, which is the Galactic value from the Planck data (Planck Collaboration XXV 2011).

For the temperature of the dust, we assume $T=25 \mathrm{~K}$. For the rest-frame frequency assumed to compute $S_{v_{\mathrm{obs}}}$, we consider $1.2 \mathrm{THz}$, corresponding to $\lambda_{\text {rest }}=250 \mu \mathrm{m}$ (see Gilli et al. 2014). At this wavelength our galaxies are expected to be optically thin based on the recent results obtained by Faisst et al. (2020a) from the SEDs of four main-sequence $z \sim 5.5$ galaxies (two contained in the ALPINE dataset), observed with ALMA in three different bands and for which constraints on the Rayleigh-Jeans part of their spectra were obtained. Our assumption is valid also considering the wavelengths at which more dusty or extreme sources than the ALPINE ones become optically thick as submillimetre galaxies (SMGs; $\lambda_{\text {rest }} \lesssim 200 \mu \mathrm{m}$, Conley et al. 2011; Riechers et al. 2013; $\lambda_{\text {rest }} \lesssim 100 \mu \mathrm{m}$, Simpson et al. 2017).

We discuss our choice of a single cold component below. Moreover, we discuss the uncertainties on the derived dust masses related to three (non-independent) factors: the bandconversion, linked to the extrapolation of the $250 \mu \mathrm{m}$ flux from higher frequencies (i.e. $157 \mu \mathrm{m}$ ); the emissivity $\beta$; and the temperature $T$.

In galaxies, dust is located primarily in two components, a warm one $(20<T<60 \mathrm{~K})$ associated with photodissociation regions (PDRs) and a cold one associated with the diffuse ISM $(T<30 \mathrm{~K}$, see Draine \& Li 2007). The adopted method relies on the assumption that the diffuse cold dust component accounts for the bulk of the dust budget. This has been shown to be true in the local Universe (see Orellana et al. 2017) where, given the high-quality photometric data, a sophisticated fit was performed showing how the contribution of the warm component accounts for a low fraction of the total dust budget $(\sim 1 \%$ and reaching the highest value of $4 \%$ for starburst galaxies). At higher redshift, up to $z \sim 2$, the validity of this approximation is advocated by Scoville et al. (2014) (see also Scoville et al. 2016, 2017) in support of the correlation between the gas masses derived from the submillimetre fluxes (directly linked to the dust masses under the optically thin approximation) and the gas masses derived from $\mathrm{CO}$ observations, under the assumption of a dust-to-gas ratio (see also the recent work of Kaasinen et al. 2019).

At the redshift of the ALPINE sources, $z \sim 5$, there is no systematic study that compares the molecular gas masses derived from the submillimetre fluxes and the $\mathrm{CO}$ measurements because the CO line is difficult to observe even with the most up-to-date interferometers. To date, far in normal main-sequence galaxies there are only two detections (D'Odorico et al. 2018; Pavesi et al. 2019). Moreover, the CO-based ALMA gas mass estimates rely on strong assumptions about the $\mathrm{CO}$ excitation mechanism to extrapolate from the high-J CO levels, accessible with ALMA, to the $\mathrm{CO}(1-0)$ fundamental line. To overcome this issue, in Dessauges-Zavadsky et al. (2020) the [CII] line is used as a tracer of the molecular gas masses for the ALPINE galaxies. By using the relation from Zanella et al. (2018), the molecular gas masses are derived from the [CII] luminosities and compared with masses inferred from dynamical masses and sub-mm fluxes under the approximation of a unique dust component at $T=$ $25 \mathrm{~K}$ and $\beta=1.8$. The good correlation shown by these three tracers (see Sect. 3 and Figs. 3 and 4 in Dessauges-Zavadsky et al. 2020) supports our assumptions. A cold dust component, dominating the dust budget, has also been considered up to very high- $z$ by Magnelli et al. (2020), taking advantage of the ALMA LP survey at 1.2mm (ASPECS, Walter et al. 2016; Decarli et al. 2019; González-López et al. 2020), where the cold component approximation is applied up to $z \sim 4.5$ for detected sources and up to $z \sim 5.5$ for stacked data (see also the 
recent review from Hodge \& da Cunha 2020 and references within).

We note that our assumed dust temperatures are massweighted mean temperatures, and should not be confused with luminosity-weighted mean temperatures, which are more closely related to the global SED shape (see Liang et al. 2019 for a detailed discussion on the different dust temperature definitions). This means that our temperatures are not comparable with the warmer temperatures obtained by Béthermin et al. (2020) by fitting the $\lambda_{\text {rest }}>40 \mu \mathrm{m}$ FIR ALPINE stacked SED ( $T=43 \pm 5 \mathrm{~K}$, see Sect. 4 in Béthermin et al. 2020) or with the dust peak temperature range $(30-43 \mathrm{~K})$ as found by Faisst et al. (2020a). Similar considerations are valid also when comparing the cold mass-weighted temperature with predictions from theoretical models. In Sommovigo et al. (2020) (but see also Narayanan et al. 2018; Liang et al. 2019) the authors show with an analytical and physically motivated model that the dust located in high- $z(>5)$ giant molecular clouds (GMCs) is warmer $(T \sim 60 \mathrm{~K})$ than it is locally since the clouds are characterised by a more compact structure than their local analogues. The warm dust located near star-forming regions has a strong radiative efficiency; therefore, it determines the shape of the SED, but it does not represent the bulk of the dust mass, expected to be at lower temperature ( $T \sim 20-30 \mathrm{~K}$, Sommovigo et al. 2020).

Considering the uncertainties related to the band conversion, we rely on the simulations performed by Privon et al. (2018). In Privon et al. (2018), the $850 \mu \mathrm{m}$ luminosities of simulated SEDs are compared with those that would have been estimated assuming a MBB approximation with $\beta=1.8$ and $T=25 \mathrm{~K}$ (as in the present analysis) under different observational set-ups. In particular, the scenario of observing only with ALMA Band 7, as in our case, has been considered (see Fig. 5 in Privon et al. 2018). The inferred luminosities are always greater than the real values, with differences of the order of $\sim 20 \%$ at $z \sim 5$ (and up to $30 \%$ for the most extreme halos). These mis-matches are due to the choice of the parameters used $(\beta$ and $T$ ) to infer the $850 \mu \mathrm{m}$ flux from data at higher frequencies, and to the fact that real SEDs are not single-temperature black bodies. We note that the results of Privon et al. (2018) are obtained at $850 \mu \mathrm{m}$ (and not at $250 \mu \mathrm{m}$ ), but are also valid in the present case. We tested that our dust masses do not change when considering 250 or $850 \mu \mathrm{m}$ and if the flux $S_{v_{\text {obs }}}$ in Eq. (1) is derived by means of an extrapolation at higher frequencies.

Considering the emissivity parameter $\beta$ and the temperature $\mathrm{T}$, we test how the dust masses would change by changing these parameters individually, and freezing the others. For the emissivity $\beta$, as stated before, we assume $\beta=1.8$, which is the Galactic value from the Planck data (Planck Collaboration XXV 2011), again in agreement with Faisst et al. (2020a). Theoretical models predict a wider range for $\beta$ (i.e. Draine 2011), and therefore we test how extreme values of $\beta=1.5(\beta=2)$ would affect our dust mass estimates, finding an increase (decrease) of the dust masses by a negligible factor of less than $\sim 15 \%$ for $\beta=1.5(\beta=2)$ (see also Magnelli et al. 2020). For the temperature of the cold dust we explore a range of values, from $T=20 \mathrm{~K}$ to $T=35 \mathrm{~K}$. We do not consider temperatures lower than $T=20 \mathrm{~K}$, given the lower limit imposed by the cosmic microwave background (at $z=5$ $6, T_{\mathrm{CMB}}=16-19 \mathrm{~K}$, respectively, see da Cunha et al. 2013). A temperature of $T=20 \mathrm{~K}$ would increase the dust masses by a factor within the uncertainties considered (20\%), while assuming $T=35 \mathrm{~K}$ would produce a decrease of the order of $60 \%$. The dust masses derived at $T=25 \mathrm{~K}$ are our fiducial values, but we discuss also the consequences of dust that is warmer by $10 \mathrm{~K}$.

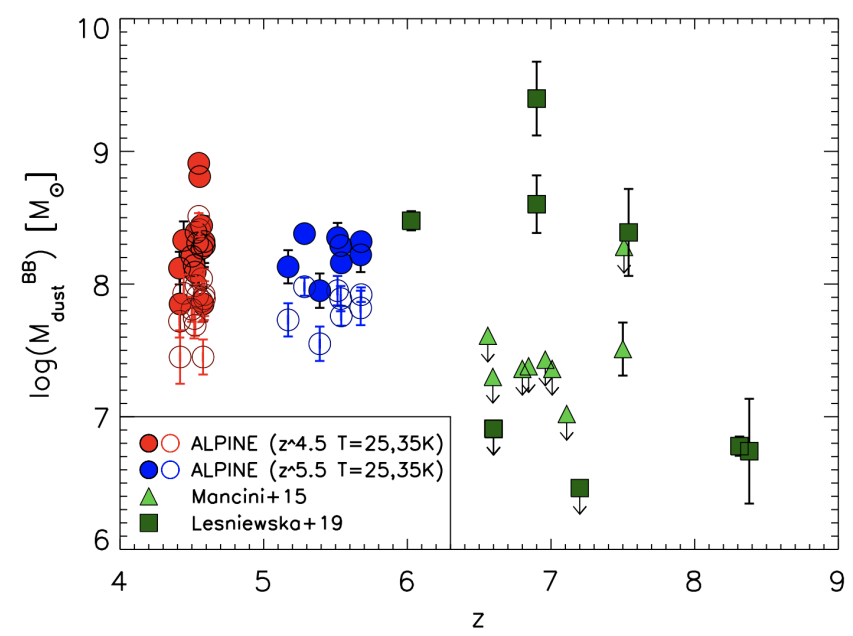

Fig. 1. Dust masses of the ALPINE continuum-detected galaxies (filled circles) plotted as a function of $z$ and computed assuming a MBB spectrum with $T=25 \mathrm{~K}$ and $\beta=1.8$ (see Sect. 3). The empty circles correspond to $T=35 \mathrm{~K}$. The blue and red points show detections at $4<z<5$ and $5<z<6$, respectively. For comparison, other compilations of dust masses at high- $z$ are shown (green triangles: Mancini et al. 2015; darkgreen squares: Leśniewska \& Michałowski 2019).

In Table A. 1 the dust masses for the 23 continuum-detected sources are reported. We calculate the error bars on the dust masses by summing in quadrature the relative uncertainties on the $157 \mu \mathrm{m}$ continuum flux $(\sim 25 \%)$, and the systematic uncertainties due to the $\beta$ emissivity $(\sim 15 \%)$ and to the band conversion emissivity $(\sim 30 \%)$. The combined errors give a typical uncertainty of $0.2-0.3 \mathrm{dex}$, expressed in $\log \mathrm{M}_{\text {dust }}$.

The dust masses of the galaxies of the ALPINE sample are shown as a function of redshift in Fig. 1 (red and blue filled circles). For most of our galaxies we find dust masses in the range $\log \left(M_{\text {dust }} / M_{\odot}\right)=7.8-8.4$, with the exception of two systems showing significantly higher values $(\log ($ Mdust $=M)=8.8$ and $\log ($ Mdust $=\mathrm{M})=8.9$ for DEIMOS_COSMOS_818760 and DEIMOS_COSMOS_873756, respectively). These two sources are the only targets presenting an ALMA continuum flux $>1 \mathrm{mJy}$ (see Table B.1. in Béthermin et al. 2020). The empty circles in Fig. 1 correspond to the estimates obtained when assuming a warmer dust temperature of $T=35 \mathrm{~K}$. In Fig. 1 we also report two collections of high- $z(z \sim 6-7)$ dust mass measurements compiled in Mancini et al. (2015) and Leśniewska \& Michałowski (2019). Altogether, the values from these works, which include both detections and upper limits, show $a \sim 3$ dex scatter, hence much larger than that obtained for our galaxies.

\section{Scaling relation of ALPINE continuum detected sources}

In this section we combine the dust masses estimates with other physical information obtained for the ALPINE targets, and we compare the observed dust scaling relations with predictions from chemical evolution models in order to understand the nature and to probe the evolution of main-sequence (MS)selected galaxies at $z \sim 4-5$. In the following section we present first a brief description of the chemical models and then the comparison of the observed dust scaling relations with the model predictions. 


\subsection{Chemical evolution models}

The chemical evolution models considered here describe galaxies with different star formation histories, namely protospheroidals (PSPHs) and spirals. The models including evolution of interstellar dust grains were presented in Calura et al. (2008) (see also Schurer et al. 2009; Pipino et al. 2011; Calura et al. 2014). Chemical evolution models calculate the evolution of the abundances of various chemical elements and dust in the ISM, generally starting from simple prescriptions to describe basic processes regulating galaxy evolution. In the past these models were extensively tested and used to interpret observational, dustrelated scaling relations in local and distant galaxies (see also Calura et al. 2017).

In the models used here, PSPHs represent the precursors of local elliptical galaxies. PSPHs form from the rapid collapse of a gas cloud of primordial composition, which triggers an intense starburst. The collapse is described by an exponential infall law, with a characteristic e-folding time depending on the mass of the galaxy (Calura et al. 2014), with typical values in the range $\sim 0.2-0.5$ Gyr (Palla et al. 2020). The starburst continues until the thermal energy of the ISM, computed as the cumulative energy deposited in the ISM by type II and type Ia SNe, equals the binding energy of the gas, computed by taking into account the gravitational contribution of the gas, the stars, and the dark matter halo, assumed to be ten times more massive than the initial gas mass (Matteucci 1994). When this occurs, star formation is immediately interrupted, and the galaxy is assumed to instantaneously eject all the residual gas. The models describe three PSPH of final baryonic mass $3 \times 10^{10} M_{\odot}, 10^{11} M_{\odot}$, and $10^{12} M_{\odot}$.

In this work we also consider a set of chemical evolution models for spiral galaxies. Each model consists of several independent rings, $2 \mathrm{kpc}$ wide, each representing a separate region of a disc. In these spiral galaxies the formation of the disc is also described by an exponential infall law, but on much longer, radius-dependent timescales, ranging from $\sim 1$ Gyr in the innermost parts up to $\sim 10 \mathrm{Gyr}$ in the outskirts, according to the inside-out scenario (Matteucci \& Francois 1989). The final stellar masses of the spirals range from $2 \times 10^{9} M_{\odot}$ to $10^{11} M_{\odot}$. In all our models, star formation is modelled by means of a Schmidt (1959) law, with a higher efficiency in more massive objects, a behaviour known as galactic downsizing (e.g., Cowie et al. 1996; Matteucci 1994; Spitoni et al. 2020).

The stellar initial mass function (IMF) assumed for spirals is that of Scalo (1986), a two-slope power-law characterised by a steeper slope $(\alpha=-1.7)$ with respect to the Salpeter value $(\alpha=-1.35)$ at stellar masses $>2 M_{\odot}$.

For the IMF of PSPH we test two different forms, the Salpeter (1955) IMF and the Larson (1998) IMF, a topheavy IMF (THIMF). The Larson IMF has the form $\phi(m) \propto$ $m^{-1.35} \exp \left(-m_{c} / m\right)$, and assuming a characteristic mass value $m_{c}=1.2 M_{\odot}$, it is top-heavy (i.e. richer in massive stars) with respect to the Salpeter IMF (see Calura et al. 2014). In all cases the IMF is assumed to be constant in time.

The use of a steeper IMF in discs is motivated by the results of Weidner \& Kroupa (2005), who have shown that since the disc stellar population is mostly made by dissolving open stellar clusters, the disc IMF must be significantly steeper than the canonical cluster IMF (with $\phi(m) \propto m^{-1.35}$ for stellar masses above $\sim 1 M_{\odot}$ ) as the former results from a folding of the latter with the star cluster mass function, which in general is a singleslope power law (e.g., Lada \& Lada 2003).

The choice of a different IMF in different models is also motivated by the requirements of reproducing the local constraints, including the abundance pattern observed in the Milky Way disc for spirals (e.g., Spitoni et al. 2019) and the abundance ratios and metal budget in local ellipticals (e.g., Calura \& Matteucci 2004; De Masi et al. 2019). For the stellar mass and SFR, the conversion between a standard IMF such as those of Chabrier (2003) and Salpeter (1955) amounts to $\sim 0.2-0.25$ dex. The same is true also when a Scalo (1986) IMF is considered. Therefore, the adoption of different IMFs produces variations which are in general much smaller than the range shown by the ALPINE sample. Overall, this implies that the impact of the choice of the IMF on the results of Fig. 2 is marginal.

The models include dust production in stars, occurring in core-collapse $\mathrm{SNe}$ and intermediate-mass stars, restoring significant amounts of dust grains during the asymptotic giant branch (AGB) phase. The set of metallicity-independent dust condensation efficiencies considered here are from Dwek (1998). Dust destruction in supernovae ( $\mathrm{SNe}$ ) shocks and dust growth in the ISM are also taken into account (see also Dayal \& Ferrara (2018) for the dust production and destruction mechanisms). The prescriptions for these processes are described in Calura et al. (2008) (see also Dwek 1998).

\subsection{Dust scaling relations}

In Fig. 2 the dust masses are reported along with other physical quantities of the ALPINE continuum-detected sources: the stellar mass $\left(M_{\text {stars }}\right)$, the star formation rate (SFR), the age, and the gas mass $\left(M_{\text {gas }}\right)$. The stellar mass, the SFR, and the age values are computed by Faisst et al. (2020b) adopting a Chabrier (2003) IMF and using the LePhare SED-fitting code (Arnouts et al. 1999; Ilbert et al. 2006). We are aware that the age is one of the most uncertain parameters of the SED-fitting analysis (i.e. Thomas et al. 2017). However, as we discuss later, the exact values found for this parameter do not significantly influence our results.

For consistency with the models, we rescale $M_{\text {stars }}$ and SFR to a Salpeter (1955) IMF by multiplying the quantities by a factor of 1.7 (see Speagle et al. 2014). For $M_{\text {gas }}$, we adopt the values from Dessauges-Zavadsky et al. (2020), obtained from the [CII] luminosities using the Zanella et al. (2018) relation, with a typical uncertainty of $\sim 0.3$ dex. In Fig. 2 we compare estimates derived for the galaxies of our sample with the results obtained from the chemical evolution models presented in Sect. 4.1. The solid circles represent the inferred quantities (red and blue for galaxies at $z \sim 4.5$ and $z \sim 5.5$, respectively), while the curves represent the PSPH and spiral models (see caption of Fig. 2 for further details).

The evolution of the dust mass as a function of age (panel a) shows that the inferred $M_{\text {dust }}$ values are in good agreement with those obtained from PSPH models at epochs comparable to the ages of the detected systems. Typical dust mass values of $\sim 10^{8} M_{\odot}$, as derived in the ALPINE sample of continuum detected sources, are achievable at early times (typically $\sim 0.1-0.5 \mathrm{Gyr}$ ) in PSPH. On the other hand, in the case of spirals, only the most massive model reaches comparable values after several Gyr of evolution (blue line in panel a), whereas the other models show much lower $M_{\text {dust }}$ values at any epoch. As previously described, this result is robust against the exact value of the ages of the ALPINE galaxies. In the extreme and unlikely case that the ages are all systematically underestimated, they cannot be larger than the age of the Universe at the redshift of our sample (vertical dashed lines in Fig. 2a).

This suggests that the PSPH models are the only ones that can cover the region occupied by the observed galaxies. The 

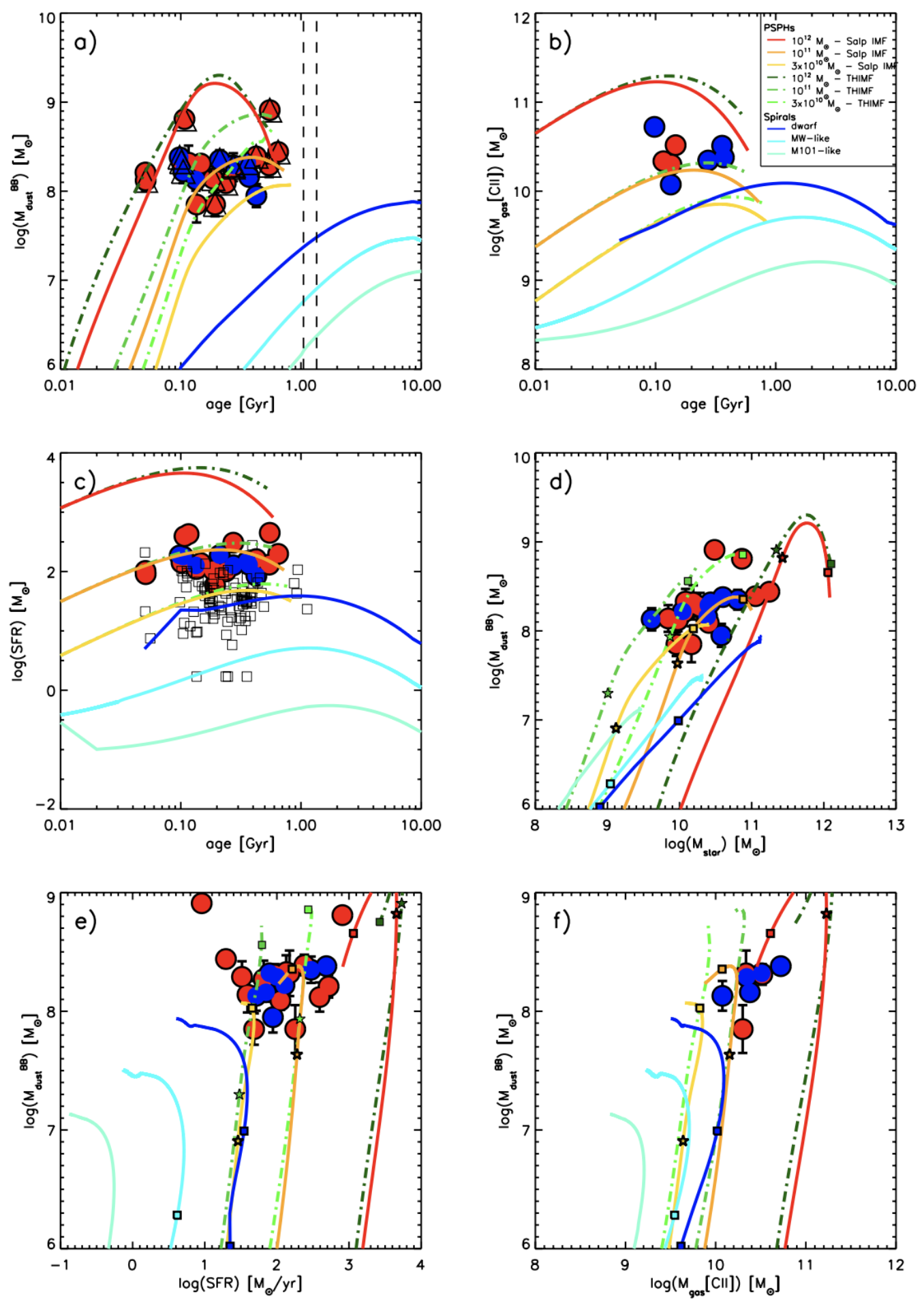

Fig. 2. Scaling relations of the ALPINE targets. Top panels: $M_{\text {dust }}$ vs. age (panel a) and $M_{\text {gas }}$ vs. age (b). Middle panels: SFR vs. age (c) and $M_{\text {dust }}$ vs. $M_{\star}(d)$. Bottom panels: $M_{\text {dust }}$ vs. SFR $(e)$ and $M_{\text {dust }}$ vs. $M_{\text {gas }}(f)$. $M_{\text {dust }}$ is computed by means of a MBB fit and assuming $T=25 \mathrm{~K}$ and $\beta=1.8$. The blue and red points show detections at $4<z<5$ and $5<z<6$, respectively. In panel $a$ the triangles around the points indicate galaxies classified as mergers or dispersion-dominated objects (Le Fèvre et al. 2020), whereas the black vertical dashed lines indicate the age of the Universe at redshift 4.5 and $z=5.5$ (corresponding to an age of the Universe of $1.35 \mathrm{Gyr}$ and $1.05 \mathrm{Gyr}$, respectively). The empty squares in panel $c$ represent the ALPINE galaxies non-detected in continuum. The curves represent the chemical evolution models described in Sect. 4.1. The yellow, orange, and red solid lines represent proto-spheroid models of baryonic mass $3 \times 10^{10}, 10^{11}$, and $10^{12} M_{\odot}$, respectively, computed with a standard Salpeter IMF (Salpeter 1955). The light green, green, and dark green dot-dashed lines are for three PSPHs with the same baryonic masses as above, but characterised by a top-heavy IMF (Larson 1998). The light cyan, cyan, and blue solid lines represent models for a dwarf spiral, an intermediate-mass spiral, and an M101-like spiral, respectively (Calura et al. 2017). The stars and squares plotted along each curve and in the same colour indicate the evolutionary times of 0.1 and $0.5 \mathrm{Gyr}$, respectively. 
other models require $>1 \mathrm{Gyr}$ to produce the observed $M_{\text {dust }}$ values. This finding is due to an overall much faster, stronger evolution shown by PSPH with respect to spirals, confirmed also by their star formation history (panel $c$ in Fig. 2). The SFR versus age plot shows that most of the SFRs measured in ALPINE galaxies are consistent with the values shown by the intermediate- and low-mass PSPH models (orange and yellow lines), with very few of them similar to the values shown by the most massive spiral models. This plot also shows a limited time interval in which the SFR values of the low-mass PSPH are very similar to those shown by the massive spirals at early times $(<1 \mathrm{Gyr})$, but in other plots these two models show quite distinct behaviours, such as in the $M_{\text {dust }}$-Age and $M_{\text {dust }}-M_{\text {stars }}$ relations. Two galaxies with particularly high $M_{\text {dust }}$ are reproduced by the PSPH models with a THIMF (green dash-dotted lines). A particularly high dust yield (defined as amount of dust per unit stellar mass, or specific dust mass) in star-forming galaxies at high redshift was found also in other works (Calura et al. 2017 and references therein), and several other reasons were proposed; in addition to the adoption of a THIMF (Gall et al. 2011), an enhanced dust accretion rate was also suggested (Pipino et al. 2011; Valiante et al. 2011).

Another explanation for this result invokes a substantial revision of our current knowledge of both dust production and accretion mechanisms, which might be needed in the case that neither destruction in $\mathrm{SNe}$ shocks nor accretion plays a significant role in regulating the dust mass budget in galaxies. In a recent analysis of the dust mass budget in local and distant starforming galaxies, Gall \& Hjorth (2018) show that the observed dust masses can be accounted for if the majority of the dust is formed by $\mathrm{SNe}$ on short timescales (i.e. during the most recent star formation episodes). This does not exclude the possibility that other processes, such as grain growth and destruction, may be at play in regulating the dust budget, but if their effects are non-negligible, they need to balance each other and occur on comparable timescales. If this is the case, several parameters related to the gas microphysics and sometimes different local thermal conditions have to be tuned to yield comparable destruction and growth rates. The most direct interpretation of this result is that stardust production is dominant with respect to other processes. In this case, a high dust yield can be accommodated assuming a THIMF. In addition, the analysis of the cold gas budget, as traced by the molecular gas content and indicated by the relation of $M_{\text {gas }}$ versus age, supports the result that ALPINE galaxies show properties mostly similar to PSPH.

A further indication supporting our conclusion is the galaxy classification of the ALPINE targets, performed by Le Fèvre et al. (2020), from a preliminary visual inspection analysis of the [CII] data cubes together with the large wealth of ancillary data (mainly HST F814W images, see Koekemoer et al. 2007, 2011). The majority of the ALPINE [CII]-detected galaxies have a disturbed morphology $(60 \%$ including mergers or extended and dispersion dominated systems) while only $13.3 \%$ show a rotating disc (the remaining $10.7 \%$ and $16 \%$ being compact or too faint to be classified). The fraction of galaxies with disturbed morphology becomes even larger $(\sim 70 \%)$ when considering only the continuum-detected sources. This does not exclude the possibility that the ALPINE galaxies might be compatible with progenitors of Milky Way (MW)-like galaxies, whose earliest assembly and star formation activity is expected to occur mostly in their spheroidal components.

Moreover, our conclusion remains robust even though it is based on only 23 ALPINE continuum detected galaxies. In
Fig. 2c, we report the SFR as a function of the age also for the ALPINE galaxies not detected in continuum (empty squares). These sources, as expected, are less extreme, characterised by a lower SFR in comparison to the continuum detected sources $\left(\langle\mathrm{SFR}\rangle \sim 56 M_{\odot} \mathrm{yr}^{-1}\right.$ and $\langle\mathrm{SFR}\rangle \sim 180 M_{\odot} \mathrm{yr}^{-1}$, respectively). Nevertheless, they still occupy a parameter space between the evolutionary tracks of the PSPH and the most massive spiral model.

In conclusion, our study of the scaling relations and our comparison with chemical evolution models indicates that ALPINE galaxies mostly show dust masses and SFR values expected in young, star-forming PSPHs. In most cases a Salpeter IMF is sufficient to account for the observed dust masses; in general, there is no particular need to invoke mechanisms such as a top-heavy IMF to enhance dust production, as seen for example in a sample of starbursts observed by Herschel (Calura et al. 2017). Our models for disc galaxies show a slower buildup of the dust mass, and at these epochs, fail to account for the observed dust masses.

Our study strongly outlines the need for statistical samples of galaxies at high- $z$ with the SED properly sampled in the FIRsub-mm part of the spectrum, in order to better derive the dust properties (mainly the temperature and the mass). This will be achieved by exploiting the synergies between sub- $\mathrm{mm} / \mathrm{mm}$ (i.e. ALMA, NOEMA) and FIR facilities, with features similar to the SPace Infrared telescope for Cosmology and Astrophysics (SPICA, Roelfsema et al. 2018) or to the Origins Space Telescope $\left(\mathrm{OST}^{1}\right)$, which together will enable a full characterisation of the spectrum from the Wien regime (FIR) up to the RayleighJeans regime (sub-)mm.

This will allow us to improve our understanding of early dust production and increase the current samples of galaxies with measured dust masses at high redshift. In the future the James Webb Space Telescope (JWST, Gardner et al. 2009), working in the near-IR and mid-IR regimes, will allow us to better constrain the stellar masses, ages, metallicities, and star formation histories of galaxies at very high redshift.

\section{Dust mass density at $z \sim 5$}

In this section we derive an estimate of the dust mass density (DMD) in the redshift range of the ALPINE survey $(4.3<$ $z<4.6$ and $5.1<z<5.9$ ). Taking into account the fact that ALPINE is a targeted survey of pre-selected galaxies, we divide the derivation of the DMD into two steps. First, we estimate the contribution of the UV-selected galaxies ( $\left.\rho_{\text {dust,UV }}\right)$, which sets a lower limit to the DMD; second, we consider the continuum serendipitously detected sources in the redshift range of the ALPINE targets, aiming to evaluate the total DMD $\left(\rho_{\text {dust,IR }}\right)$.

\subsection{Contribution to the DMD from UV-selected galaxies}

The ALPINE targets are UV-selected sources, with UV magnitude $L_{\mathrm{UV}}>0.5 L^{\star}$ (see Sect. 2). Among the 118 ALPINE targets, 23 sources $(\sim 20 \%$ of the sample) are FIR continuum detected and, as a consequence, to derive the average properties of the parent UV-selected population we also need to take into account the contribution of the non-detected galaxies. To this end, we performed a stacking analysis on the ALMA maps.

We perform stacking using the $\lambda_{158 \mu \mathrm{m}}$ rest-frame continuum images centred on the UV counterpart positions, including both individual detections and non-detection in order to avoid biases. The procedure used is extensively described

http://origins.ipac. caltech.edu 
Table 1. Results of the stacking analysis.

\begin{tabular}{lccc}
\hline \hline Redshift & $N$ & $\begin{array}{c}\log L_{\mathrm{UV}} \\
{\left[L_{\odot}\right]}\end{array}$ & $\begin{array}{c}\log M_{\text {dust }} \\
{\left[M_{\odot}\right]}\end{array}$ \\
\hline \multirow{2}{*}{$4.4<z<4.6$} & $23(2)$ & $10.4-10.9$ & $7.53_{-0.17}^{+0.15}$ \\
& $23(8)$ & $10.9-11.1$ & $7.83_{-0.14}^{+0.13}$ \\
& $21(5)$ & $11.1-11.6$ & $7.83_{-0.14}^{+0.11}$ \\
\hline $5.1<z<5.9$ & $25(0)$ & $10.4-10.9$ & $<7.5$ \\
& $10(1)$ & $10.9-11.1$ & $<7.6$ \\
& $15(7)$ & $11.1-11.6$ & $7.72_{-0.2}^{+0.2}$ \\
\hline
\end{tabular}

Notes. Median $M_{\text {dust }}$ are reported, derived from the median stacked fluxes obtained as described in Sect. 5.1 and using Eq. (1) as for the targets. The upper limits are at $3 \sigma$. The number of sources stacked in each bin and individually detected are also reported.
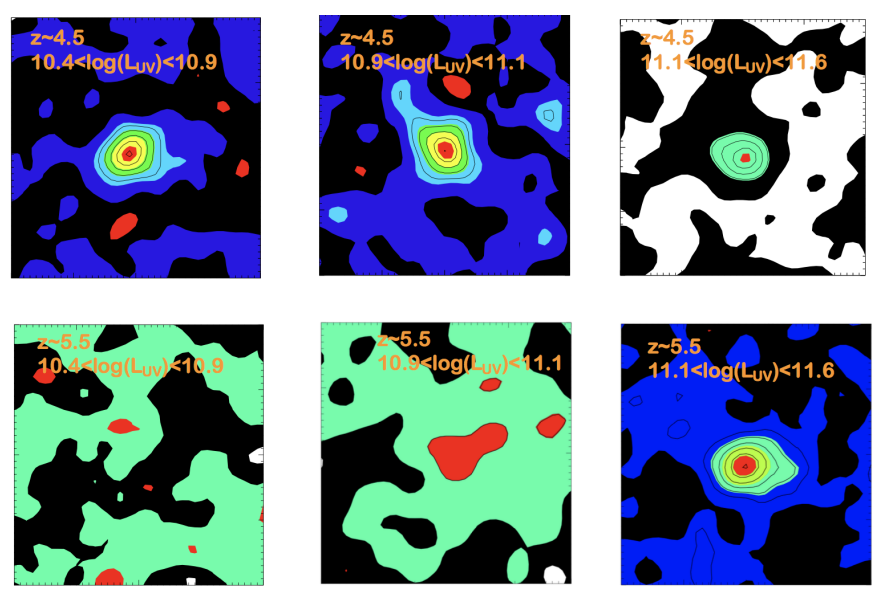

Fig. 3. $6^{\prime \prime} \times 6^{\prime \prime}$ cutouts of $L_{\mathrm{UV}}$ binned stacks of ALMA continuum images used to derive the stacked dust masses. The upper and lower panels show stacks of galaxies at $z \sim 4.5$ and $z \sim 5.5$, respectively. The black solid contours show $2,3,4,5 \sigma$. In the $z \sim 4.5$ bins all stacks have a clear detection at $>4 \sigma$, but in the $z \sim 5.5$ bins the stacks have a clear detection only at the highest $L_{\mathrm{UV}}$ bin. In Table 1 the results of the stacking analysis are summarised, including the number of sources stacked in each bin of $L_{\mathrm{UV}}$.

in the ALPINE paper of Khusanova et al. (2021) (see also Fudamoto et al. 2020a). Here we briefly summarise the main points. We use median stacking on the UV counterpart positions of both detected and non-detected objects. For the detections we adopt a $3.5 \sigma$ threshold. In the case of non-detections we use conservative $3 \sigma$ upper limits by adding three times the background RMS to the local maximum (see Béthermin et al. 2020) We perform the stacking in two redshift bins, at $z<5$ and $z>5$, and we further split the sample in bins of UV luminosity. The bins are chosen to have an almost equal number of sources in each inter$\operatorname{val}(\sim 20)$. In Table 1 the results of our stacking analysis are summarised, while in Fig. 3 the stacked images are shown. From the stacks at $z<5$, we detected significant continuum emission from all the $L_{\mathrm{UV}}$ bins (Fig. 3, upper panels), while from the stacks at $z>5$ we detected continuum emission only in the highest $L_{\mathrm{UV}}$ bin (Fig. 3, lower panels).

In Fig. 4 the dust masses as a function of their UV luminosity are shown for the continuum detected sources (23 objects), for the non-detections, and for the stacked images. For the nondetections and the stacked images the same procedure adopted for the detected sources was used to estimate the dust masses,

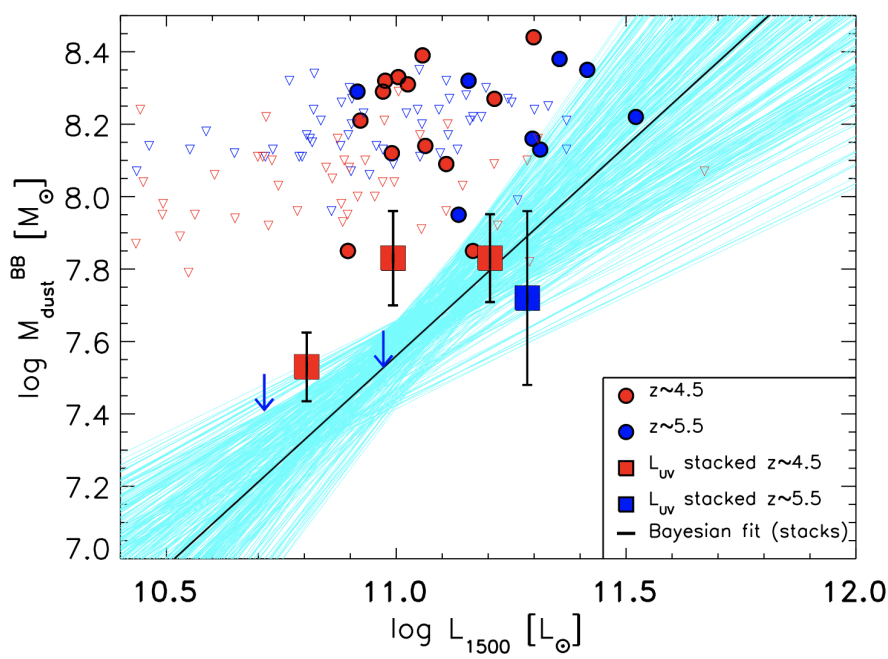

Fig. 4. $M_{\text {dust }}$ vs. $L_{\mathrm{UV}}$ for the ALPINE targets. Blue and red points show individual FIR continuum detections at $4<z<5$ and $5<z<6$, respectively. Open downward triangles show $3 \sigma$ upper limits of individual IR non-detections. Stacks with detections at $z<5$ and $z>5$ are shown as blue and red squares, respectively. The non-detection of stacks are indicated by a downward arrows. $M_{\text {dust }}$ from the stacked ALMA continuum fluxes were estimated using the same expression as for the targets (Eq. (1)). The Bayesian linear fit of all the stacked values (detections and upper limits) is shown by the black and light blue lines, which also illustrate the probability distribution of the fit at $1 \sigma$ confidence level. The resulting best fit is $\log \left(M_{\text {dust }}\right)=\left(1.16_{-0.57}^{0.68}\right)\left(\log \left(L_{\mathrm{UV}}\right)-11.\right)+$ $\left(-0.11_{-0.15}^{0.10}\right)+7.76$ (dispersion $\sigma_{\sim}^{<} 0.2$ dex, Pearson rank coefficient of 0.76 and $p$-value 0.08 , with the Pearson coefficient computed considering all data as detections).

considering the $3 \sigma$ flux upper limits and the stacked results as rest-frame $158 \mu \mathrm{m}$ continuum fluxes, respectively. The black line represents the $\operatorname{linear} \log \left(M_{\text {dust }}\right)-\log \left(L_{\mathrm{UV}}\right)$ relation obtained using the stacked values. We performed the fit using the method described in Feldmann (2019) to evaluate the likelihood in the presence of censored data. This method is a generalisation of the statistical approach of Kelly (2007), and is implemented in the LeoPy python package. The evaluation of the likelihood was combined with a Bayesian tool to realise the Markov chain Monte Carlo (MCMC) distribution for parameter space exploration, implemented in the emcee python package (Foreman-Mackey et al. 2013). The fit yields a poor significance level, with the coefficient constrained at $1-2 \sigma$ (Pearson rank coefficient of 0.76 and $p$-value of 0.08 , estimated considering all data as detections). Regardless of the poor significance of the fit, we are confident about the $L_{\mathrm{UV}}-M_{\mathrm{dust}}$ correlation, as a secondary effect of the relation between SFR and $M_{\text {dust }}$, in galaxies where $L_{\mathrm{UV}}$ captures most of the SFR (as expected for the ALPINE targets, characterised generally by low extinction values, $\langle(E-V)\rangle \sim 0.08$; see Faisst et al. 2020b).

The obtained relation allows us to derive the DMD for the UV-selected sources. In particular, since the ALPINE sample is not a volume-limited sample, to derive the comoving density of the physical property $\left(M_{\text {dust }}\right)$ we use $L_{\mathrm{UV}}$ as proxy of $M_{\text {dust }}$, following the same approach described in a companion ALPINE paper (Khusanova et al. 2021) where the authors derived the SFRD starting from the continuum detected ALPINE UV-selected sources.

Following Khusanova et al. (2021) (see also Fontana et al. 2004; Pozzi et al. 2020), we estimate the comoving density of the physical property by convolving the volume density of the 
Table 2. Cosmic dust mass density derived from the ALPINE survey at $z \sim 5$.

\begin{tabular}{lc}
\hline \hline \multicolumn{2}{c}{$\log \left(\rho_{\text {dust }}\right) z \sim 5$} \\
\hline $\log \left(\rho_{\text {dust,UV }}\right)$ & $\log \left(\rho_{\text {dust,IR }}\right)$ \\
{$\left[M_{\odot} \mathrm{Mpc}^{-3}\right]$} & {$\left[M_{\odot} \mathrm{Mpc}^{-3}\right]$} \\
\hline $4.8_{-1.0}^{+0.4}$ & $5.3_{-0.3}^{+0.5}$ \\
$>4.3$ & $>4.8$ \\
\hline
\end{tabular}

Notes. The left column lists the measurement derived from the ALPINE UV-selected galaxies using $L_{\mathrm{UV}}$ as proxy for $M_{\text {dust }}$ and integrating the UVLF in the typical $0.03 L^{\star}-100 L^{\star}$ range. The right column lists the measurement derived from the serendipitous IR-detected sources using $L_{\mathrm{IR}}$ as proxy for $M_{\text {dust }}$ and integrating the IRLF in the typical $9<\log \left(L_{\mathrm{IR}}\right)<14$ range. We also report in the second row the lower limits obtained by integrating the UVLF and IRLF only in the range sampled by the detected sources $\left(10.4<\log \left(L_{\mathrm{UV}}\right)<11.5\right.$ and $11.2<\log \left(L_{\mathrm{IR}}\right)<13$, respectively).

proxy with the mean $M_{\text {dust }}-L_{\mathrm{UV}}$ relation as

$\rho_{\text {dust }, \mathrm{UV}}=\int\left\langle M_{\mathrm{dust}}\right\rangle(x) \phi(x) d x$,

where $x$ is $L_{\mathrm{UV}}$ and $\phi(x)$ is the UV luminosity function (UVLF). In this operation we convert the mean relation found in log space (see Fig. 4) to the mean relation in linear space as

$\left\langle M_{\text {dust }} / L_{\mathrm{UV}}\right\rangle=10^{\left(\left\langle\log \frac{M_{\text {dust }}}{L_{\mathrm{UV}}}\right\rangle+\frac{2.3 \times \sigma^{2}}{2}\right)}$,

where $\sigma$ is the dispersion of the data in log space as obtained by the Bayesian fit ( $\sigma \sim 0.2$ dex, see Fig. 4).

Two main factors could affect our method and should be mentioned. First, we consider the ALPINE targets as a fair representative sample of the UV sources; second, we extrapolate the $\log \left(M_{\text {dust }}\right)-\log \left(L_{\mathrm{UV}}\right)$ relation found for $10.4<\log \left(L_{\mathrm{UV}}\right)<11.5$ to a broader $L_{\mathrm{UV}}$ range. Considering the first issue, we rely on Faisst et al. (2020b) (their Fig. 17), who show how the ALPINE sources occupy the same region of the parent sample in COSMOS (rest-frame UV-optical selected at $z \sim 5$ ) in the $M_{\text {star }}-$ SFR plane, leading the authors to conclude that the ALPINE sources are a fair representation of UV-selected star-forming galaxies at $z>4$. This is not intended to suggest that the ALPINE galaxies are a representation of all star-forming galaxies since we are aware that the UV selection does not take into account ultradusty galaxies. We discuss this point in Sect. 5.3. For the second issue we estimate two quantities, the dust mass density derived by integrating the UV luminosity only over the ALPINE UV luminosity range, and the dust mass density obtained by extrapolating the relation down to fainter UV luminosity not sampled by the ALPINE targets, and integrating over the commonly used $0.03 L^{\star}-100 L^{\star}$ range $\left(\rho_{\text {dust }, \mathrm{UV}}\right)$.

Given the procedure described above, we estimate the uncertainty on $\rho_{\text {dust,UV }}$ combining the uncertainties affecting the UVLF determination and the $\log \left(M_{\text {dust }}\right)-\log \left(L_{\mathrm{UV}}\right)$ relation. At $z \sim 5$, the UVLF is quite well constrained, the main uncertainty being the faint-end slope $\alpha$ (i.e. Bouwens et al. 2015; Ono et al. 2018; Khusanova et al. 2020). We use the derivation from Ono et al. (2018) and we estimate the associated uncertainty by varying each of the UVLF parameters $\left(\alpha, L_{\star}\right.$, and $\left.\Phi_{\star}\right)$ within $1 \sigma$ (see Table 7 in Ono et al. 2018). We find an uncertainty of $\sim 0.2$ dex in $\rho_{\text {dust,UV }}$, of the same order as the uncertainties derived from the $1 \sigma$ dispersion of the $\log \left(M_{\text {dust }}\right)-\log \left(L_{\mathrm{UV}}\right)$ relation.

The results are reported in Table 2.

\subsection{Contribution to the DMD from FIR blind detected galaxies}

In the previous section, by integrating the UVLF over the wide $0.03 L^{\star}-100 L^{\star}$ range, we have estimated the value of the DMD of the population of rest-frame UV-selected galaxies. In this section we estimate the DMD from the population of rest-frame FIR-selected galaxies. To this end, we consider the blind continuum non-target detections in the ALPINE survey in the redshift range corresponding to the ALPINE targets $(4.1<z<5.9)$ (see Sect. 2). For these sources we estimated the dust masses as was done for the ALPINE targets. We now consider $L_{\mathrm{IR}}$ as a proxy of $M_{\text {dust }}$, and as luminosity function proxy the recent determination from Gruppioni et al. (2020). Of the ten sources blindly detected, five sources were found to have a spectroscopic redshift (from the detection of the [CII] emission line) very close to the ALPINE central targets (see Sect. 3.2 in Gruppioni et al. 2020). Since these sources are possibly related to an overdensity (see also Lemaux et al. 2018; Loiacono et al. 2021 on this issue), we consider the conservative IRLF determination from Gruppioni et al. (2020), where these sources have been removed. For $L_{\mathrm{IR}}$ we consider the values measured with the SED obtained from stacking the photometric data points of the ALPINEanalogue galaxies in the COSMOS field from $\lambda_{\text {rest }}>40 \mu \mathrm{m}$. In Fig. 5 (top) the values of $M_{\text {dust }}$ as a function of $L_{\mathrm{IR}}$ are reported together with the best-fitting relation. The fit results in a high level of significance (Pearson rank coefficient 0.97 and $p$-value of $10^{-5}$ ).

As for the DMD obtained for the UV-galaxy population, we estimate the comoving density of our physical parameter ( $\rho_{\text {dust,IR }}$, where now the subscript $I R$ stands for derived from IRselected sources) by convolving the volume density of the proxy $\left(L_{\mathrm{IR}}\right)$ with the $M_{\text {dust }}-L_{\mathrm{IR}}$ relation as

$\rho_{\text {dust,IR }}=\int\left\langle M_{\text {dust }}\right\rangle \phi(x) \mathrm{d} x$,

where now $x$ is $L_{\mathrm{IR}}$ and $\phi(x)$ is the IR-luminosity function (IRLF). As was done for $L_{\mathrm{UV}}$, to convert the mean relation from $\log$ space to linear space we take into account the dispersion, although given the small dispersion $(\lesssim 0.05 \mathrm{dex})$, the second term in Eq. (3) is negligible.

For $\rho_{\text {dust,UV }}$ and also for $\rho_{\text {dust,IR }}$ we consider two quantities: a lower limit obtained from integrating the IRLF only in the IR luminosity range sampled by the data $\left(11.7<\log \left(L_{\mathrm{IR}}\right)<13\right)$, and the value $\left(\rho_{\text {dust,IR }}\right)$ obtained from integrating the IRLF in the commonly used IR range $\left(\rho_{\text {dust,IR }} ; 9<\log \left(L_{\mathrm{IR}}\right)<14\right.$, see Gruppioni et al. 2020).

The uncertainty on $\rho_{\text {dust,IR }}$ depends on the uncertainties affecting the IRLF and on the uncertainties affecting the $\log \left(M_{\text {dust }}\right)-\log \left(L_{\mathrm{IR}}\right)$ relation. The former was computed considering the $1 \sigma$ uncertainty derived through the MCMC analysis of the IRLF estimated by Gruppioni et al. (2020) (see their Table 4). This term accounts for a $1 \sigma$ dispersion of $\sim 0.3-0.5$ dex in $\rho_{\text {dust,IR }}$, significantly higher than that derived from the dispersion of the $\log \left(M_{\text {dust }}\right)-\log \left(L_{\mathrm{IR}}\right)$ relation $(\lesssim 0.05$ dex $)$.

The results are reported in Table 2.

\subsection{Discussion on the DMD derivation}

In Fig. 6 we show the evolution of the comoving DMD as a function of the look-back time from our analysis, together with determinations from other observational works and with model predictions. The ALPINE derivations are reported at $12<t_{\text {lookback }}<$ $12.5 \mathrm{Gyr}$, corresponding to the redshift range of the ALPINE 

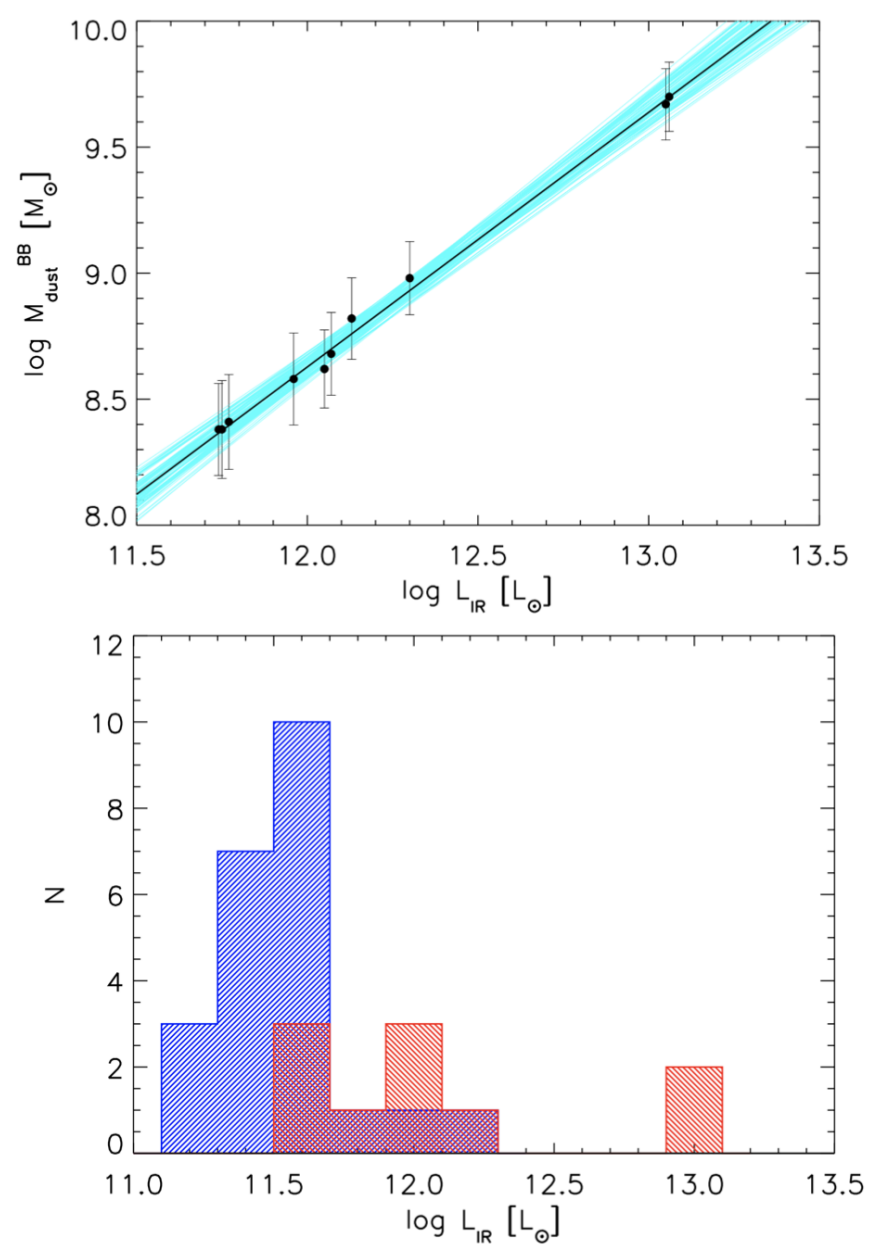

Fig. 5. Properties of the non-target serendipitously detected sources. Top: $M_{\text {dust }}$ vs. $L_{\mathrm{IR}}$ for the non-target serendipitously detected sources in the ALPINE redshift range $(4.1<z<5.9)$. The corresponding $M_{\text {dust }}$ was calculated using the same expression as for the ALPINE targets (Eq. (1)). The Bayesian linear fit is shown by the black and light blue lines, which also illustrate the probability distribution of the fit at $1 \sigma$ confidence level. The resulting best fit is $\log \left(M_{\text {dust }}\right)=$ $\left(1.01_{-0.11}^{0.11}\right)\left(\log \left(L_{\mathrm{IR}}\right)-12.2\right)+\left(-0.0_{-0.05}^{0.05}\right)+8.8$ (dispersion $\sigma_{\sim}^{<0.05} \mathrm{dex}$, Pearson rank coefficient of 0.97 , and $p$-value $10^{-5}$ ). Bottom: distribution of the $L_{\mathrm{IR}}$ for the target continuum detected (blue histogram, $\left.\left\langle\log \left(L_{\mathrm{IR}}\right)\right\rangle=11.6_{-0.2}^{+0.1}\right)$ and for the serendipitously detected (red histogram, $\left.\left\langle\log \left(L_{\mathrm{IR}}\right)\right\rangle=12.1_{-0.3}^{+0.9}\right)$ ALPINE sources. $L_{\mathrm{IR}}$ valuees were estimated using the stacked SED from ALPINE analogues in the COSMOS field from Béthermin et al. (2020).

sources (for the case of the derivation from the UV populations a slightly wider range is considered for display purposes). The values obtained from the UV-selected population (IR-selected population), obtained by integrating the UVLF (IRLF) in the extended UV (IR) luminosity range are represented by blue (red) points. The boxes around the points represent the $1 \sigma$ associated uncertainties, with the large dispersion of $\rho_{\text {dust,UV }}$ reflecting the large dispersion in the $\log \left(M_{\text {dust }}\right)-\log \left(L_{\mathrm{UV}}\right)$ relation (see Fig. 4$)$.

The value of $\rho_{\text {dust,UV }}$ is $\sim 30 \%$ of $\rho_{\text {dust,IR }}$ (although consistent within $1 \sigma$ ), and this is not surprising since the UV-selected population is not representative, by construction, of the most dusty obscured galaxies which are present in the blind IR selection. This is supported by the comparison of the $L_{\mathrm{IR}}$ distribution of the ALPINE targets and of the serendipitously detected sources. The serendipitous sources show $\left\langle\log \left(L_{\mathrm{IR}}\right)\right\rangle=12.1_{-0.3}^{+0.9}$, hence they are generally brighter than the ALPINE targets, with $\left\langle\log \left(L_{\mathrm{IR}}\right)\right\rangle=$ $11.6_{-0.2}^{+0.1}$ (see Fig. 5, bottom). While this difference alone is not conclusive since it could be partially caused by different $S / N$ cuts $(S / N=3.5$ and 5 , for our targets and the serendipitous sources, respectively, see Béthermin et al. 2020), in addition to their different IR luminosity distributions, the fact that only half of the serendipitous sources (see Gruppioni et al. 2020) are detected in the UV at magnitudes $z \sim 25.9$ (Laigle et al. 2016) further supports our hypothesis.

Our result is also in line with that found recently by Gruppioni et al. (2020) for the SFRD. By deriving the SFRD from IR sources serendipitously detected in ALPINE, the authors found that the difference with literature UV results increases with redshift, reaching a factor $\sim 10$ at $z \sim 6$.

In Fig. 6 we also show previous determinations of $\rho_{\text {dust }}$ (see references in the figure legend and caption). In the redshift range sampled by the ALPINE survey, recent determinations were derived by Péroux \& Howk (2020) and Magnelli et al. (2020). Péroux \& Howk (2020) (orange solid squares in Fig. 6) estimated the DMD indirectly, by convolving the mass density of neutral gas with the dust-to-gas (DTG) ratio. The density of neutral gas was derived from an extensive literature collection of different measurements, mainly based on high- $z$ quasar spectral absorption features, whereas the DTG was estimated from the depletions of different heavy elements into the solid phase. The determinations from Péroux \& Howk (2020) are consistent with our $\rho_{\text {dust }}$ value obtained from UV-selected galaxies, whereas it is lower than our fiducial value derived from IRselected galaxies. However, Péroux \& Howk (2020) cautioned the reader against a potential selection bias affecting their estimate (see their Sect. 3.2.3), as extremely dusty systems might be missing from their optically selected quasar sample. The large uncertainty (up to $50 \%$ ) affecting our determination of $\rho_{\text {dust }}$ for IR-selected galaxies reflects the small sample used to estimate the IRLF. This further points out the need for future investigations and for future space IR high-sensitivity instruments suitable for tracing the dust content of high-redshift galaxies.

Magnelli et al. (2020) estimate the DMD directly, thanks to ALMA observations from the ASPECS LP survey (yellow solid squares). The ASPECS LP survey (González-López et al. 2020) was obtained in Band 6 (1.2 mm), over an area of $\sim 4.2 \mathrm{arcmin}^{2}$. The reported determinations from the ASPECS survey from $0.3<z<5.5$ were computed by stacking the ALMA maps at the positions of $H$ band-selected galaxies above a stellar mass of $10^{8} M_{\star}$ in distinct redshift bins. The points corresponding to $3.2<z<4.5(4.5<z<5.5)$ were obtained by stacking 44 (9) galaxies, but since the sample cannot be considered stellar mass complete, these measurements can only be considered lower limits (see Table 1 in Magnelli et al. 2020). Our values of $\rho_{\text {dust }}$ obtained from the UV-selected and the FIR-selected galaxies, at the redshift of the ALPINE survey $(4.5<z<5.9)$, are consistent with the lower limits from the ASPECS survey obtained in the two highest redshift bins $(3.2<z<4.5$ and $4.5<z<5.5)$.

The general DMD as a function of cosmic time shows a mild increase from $z \sim 5$ up to cosmic noon $(1<z<3$ ) (a factor of $\sim 2.5$ in our analysis), followed by a smooth decline (a factor of $\sim 3$ ) up to the local Universe (see also Driver et al. 2018; Pozzi et al. 2020; Magnelli et al. 2020).

By comparing the DMD and the SFRD, as already stated by Magnelli et al. (2020), at $z<2$ both quantities show a decline toward the local Universe, but the decline of the DMD is less pronounced than that observed for the SFRD (a factor $\sim 3-4$ instead of $\sim 8$ ). At $z>3$, the SFRD derived from IR data shows 


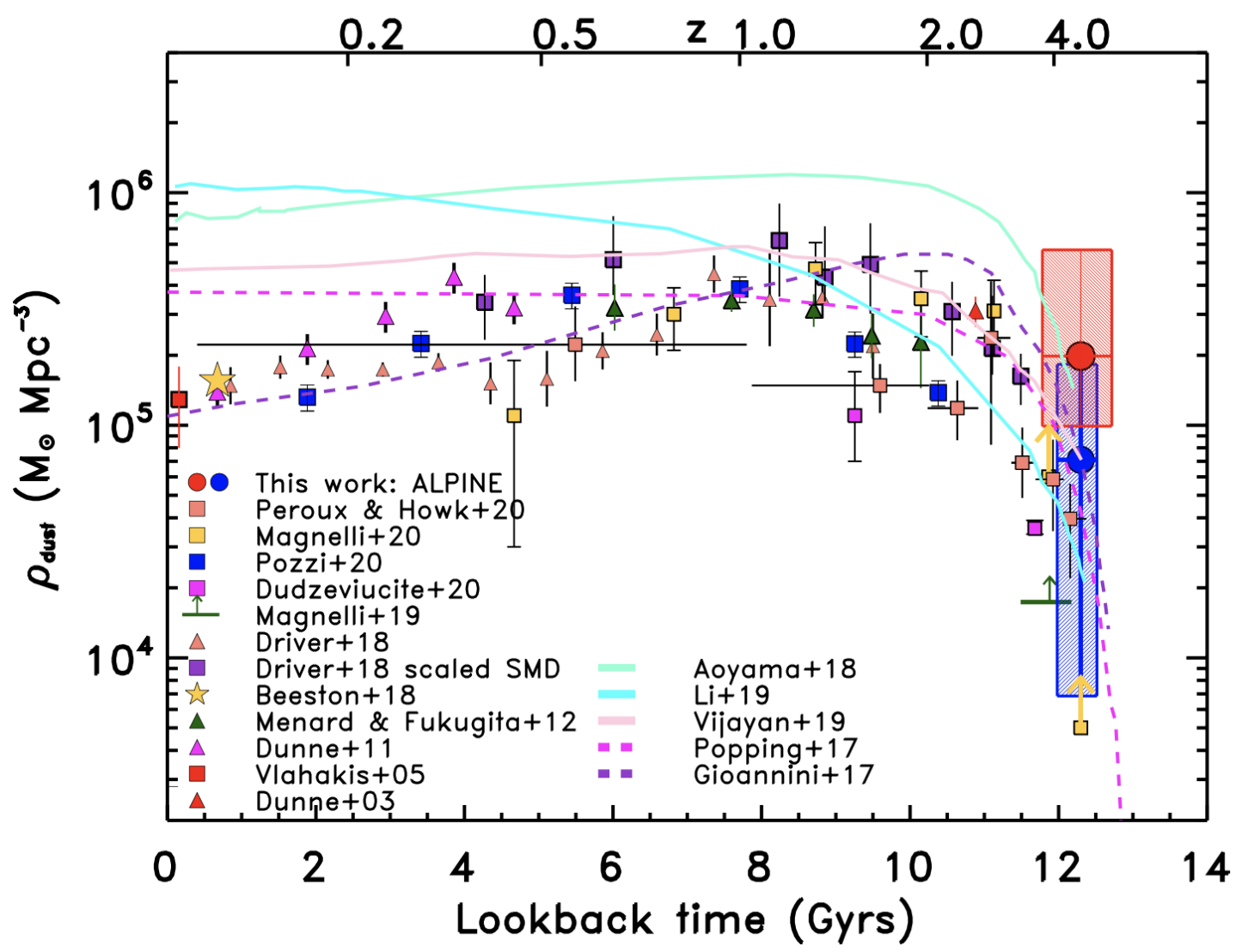

Fig. 6. Evolution of the comoving dust mass density $\left(\rho_{\text {dust }}\right)$ as a function of the look-back time. Our determinations are shown at $t_{\text {lookback }} \sim 12.3$ Gyr, obtained from the ALMA continuum detection of the UV-selected (blue circle) and serendipitously detected sources (red circle), respectively. The blue and red boxes represent the $1 \sigma$ uncertainties. For comparison, estimates from other surveys are shown (ALMA: Magnelli et al. 2020; Herschel: Dunne et al. 2011; Driver et al. 2018; Beeston et al. 2018; Pozzi et al. 2020; SCUBA: Dunne et al. 2003; Vlahakis et al. 2005; Dudzevičiūte et al. 2021; IRAM: Magnelli et al. 2019; absorbers in the optical spectra of quasars: Ménard \& Fukugita 2012; Péroux \& Howk 2020). The lines represent different models: the cosmological hydrodynamic models from Aoyama et al. (2018) and Li et al. (2019) (light green and cyan, respectively); the semi-analytical models from Popping et al. (2017) and Vijayan et al. (2019) (magenta dashed and pink solid lines, respectively); the chemical evolution model from Gioannini et al. (2017) (dark green dashed line).

an almost flat behaviour (Gruppioni et al. 2020 and references within), while the DMD shows a mild decline (a factor of 2.5 ). We postpone the interpretation of these data to a future theoretical work. Nevertheless, we note that the cosmic dust mass at $z \sim 5$ may not have reached its maximum value yet, and this could be partially explained by considering the typical dust production timescales, the time after which the dust mass reaches its maximum in galaxies, which ranges from a few $0.1 \mathrm{Gyr}$ up to several Gyr in spirals and proto-spheroids, respectively (see panel a in Fig. 2).

In Fig. 6 we also show the predictions from models. The model from Gioannini et al. (2017) is the one that best reproduces the global trend shown by the DMD evolution from the local Universe up to $z \sim 5$. This is not a cosmological model, but a phenomenological one that combines the chemical evolution of different galaxy types (Calura et al. 2008) with the evolution of different galaxies as derived from Pozzi et al. (2015), based on the IRLF from Gruppioni et al. (2013). The results of Popping et al. (2017) and Vijayan et al. (2019) were obtained by means of cosmological, semi-analytical galaxy formation models. They both roughly account for the observed decrease in the DMD at high- $z(z>3)$, but they fail to reproduce the decline at lower $z$ and they overestimate the dust mass budget detected locally in resolved galaxies.

On the other hand, the models from Aoyama et al. (2018) and Li et al. (2019) are based on cosmological hydrodynamic simulations. They are globally inconsistent with the data, severely overestimating the DMD at $z<1$. At higher redshift the model from Aoyama et al. (2018) is in approximate agreement with the observations, whereas the model from Li et al. (2019) underestimates our fiducial DMD by up to a factor of $\sim 10$.

Clearly the DMD at high redshift needs further investigation from both an observational and a theoretical point of view. On the observational side, we note that the DMD is estimated assuming our fiducial temperature of $T=25 \mathrm{~K}$, a choice also made in several other studies (e.g., Scoville et al. 2014; Magnelli et al. 2020). As stated in Sect. 3, a warmer dust mass $(T=35 \mathrm{~K})$ would reduce the DMDs by a factor of $60 \%$.

In order to shed more light on the trend of the DMD at $z>2$, particularly in relation to the cosmic SFRD, a significant step forward will be achieved from the synergy of FIR and (sub-)mm facilities, allowing the consideration of at least two dust components (warm and cold dust). This will be possible when the ALMA data are complemented by data from a future FIR satellite similar to SPICA, with a sensitivity more than an order of magnitude better than Herschel (Roelfsema et al. 2018).

\section{Summary}

We used observations of the ALPINE survey to study the dust mass content of normal star-forming galaxies at high redshift 
$(z \sim 5)$ and to provide, for the first time, an estimate of the cosmic dust mass density (DMD) at such high look-back time.

ALPINE is a targeted survey specifically designed to detect the bright $[\mathrm{CII}] 158 \mu \mathrm{m}$ line in $118 \mathrm{UV}$-selected galaxies in the redshift range $4.4<z<5.9$. For the aims of the present analysis we considered the rest-frame FIR continuum emission of the ALPINE galaxies (individually detected or their stacks) and the FIR continuum emission of the blind serendipitously detected galaxies in the ALPINE area.

Dust masses were measured from the continuum emission at $250 \mu \mathrm{m}$ (see Gilli et al. 2014), extrapolated from the $157 \mu \mathrm{m}$ ALMA data, assuming a mass-weighted temperature of $25 \mathrm{~K}$ and a dust emissivity of $\beta=1.8$ Our main results can be summarised as follows:

- We combined the dust masses of the 23 ALPINE continuum detected sources with other robustly determined physical parameters derived from SED fitting (i.e. Age, $M_{\star}$, SFR) or from the ALPINE observations themselves (i.e. $M_{\text {gas }}$ ) to settle the evolutionary stage of normal star-forming galaxies at $z \sim 5$. For this purpose, we compared the observed dust scaling relation with the evolutionary tracks predicted by chemical models for spiral galaxies and for precursors of local elliptical galaxies of different masses (called proto-spheroids, or PSPHs). From the analysis of several scaling relations, we conclude that our sample of galaxies show dust masses and SFR values typically consistent with intermediate- and low-mass PSPH models. Our models indicate that at early epochs, galaxy discs such as that of the MW show SFR values $<10 M_{\odot} \mathrm{yr}^{-1}$ and dust masses that are much lower than those measured in ALPINE galaxies. Our conclusion is also confirmed by the non-detected galaxies, which, even if less extreme than the actual detections, in the SFRAge diagram generally show SFR values compatible with those of the massive discs, which, in the galactic downsizing picture, at these epochs are expected to be more intensely star-forming than low- and intermediate-mass spirals. It is worth noting that our results do not exclude the possibility that the ALPINE galaxies might be compatible with the progenitors of MW-like galaxies, whose earliest assembly and star formation activity is expected to occur mostly in their spheroidal components, in their bulges (e.g., Calura et al. 2012; Roca-Fàbrega et al. 2016; Fragkoudi et al. 2020).

- We estimate $\rho_{\text {dust }}$ at $z \sim 5$ for UV-selected and FIR-selected galaxies, using $L_{\mathrm{UV}}$ and $L_{\mathrm{IR}}$ as proxies for $M_{\text {dust }}$, respectively. In the first case we use the $\log \left(M_{\text {dust }}\right)-\log \left(L_{\mathrm{UV}}\right)$ relation found for the ALPINE galaxies (considering both the detected and the non-detected sources) and the convolution with the UVLF; in the second case we consider the $\log \left(M_{\text {dust }}\right)-\log \left(L_{\mathrm{IR}}\right)$ relation found for the ten blindly FIRdetected sources and the convolution with the IRLF. The derived $\rho_{\text {dust,UV }}$ is $\sim 30 \% \rho_{\text {dust,IR }}$, although the two estimates are marginally consistent at $1 \sigma$. Our result supports the conclusion that UV-selected galaxies miss the most obscured and/or dusty objects and we consider $\rho_{\text {dust,IR }}$ as our fiducial value.

- We compare our fiducial $\rho_{\text {dust }}$ at $z \sim 5$ with the predictions from models, also taking into account the $\rho_{\text {dust }}$ determinations at lower $z$ from the literature. The phenomenological model from Gioannini et al. (2017) is the model that best reproduces the observed evolution of $\rho_{\text {dust }}$. Since this model is basically built upon the observed evolution of the cosmic SFR, this result indicates that it can roughly account for the expected timescales for dust production in galaxies. On the other hand, the ab initio cosmological, semi-analytical models of Popping et al. (2017) and Vijayan et al. (2019) and the cosmological simulations of Li et al. (2019) approximately account for the observed evolution of $\rho_{\text {dust }}$ dust at $z>1$, but they overestimate the dust mass budget at lower redshift, failing to reproduce the decreasing trend observed at $z<1$. These results indicate that our physical understanding of the cosmic evolution of the dust mass needs to be improved.

Our study strongly highlights the need for larger statistical samples of galaxies at high redshift with a full characterisation of the IR spectrum, from the Wien regime up to the Rayleigh-Jeans regime. This can be achieved by exploiting the synergies between (sub-)mm (i.e. ALMA, NOEMA) and FIR facilities.

Acknowledgements. This paper is dedicated to the memory of Olivier Le Fèvre, PI of the ALPINE survey. We are grateful to an anonymous referee for valuable suggestions that improved the paper. F. P. thanks Simone Bianchi for useful discussion on the dust properties. We acknowledge support from grant PRIN MIUR 2017- 20173ML3WW_s. F. P. also acknowledges support from the INAF man-stream program 'Gas-DustPedia: A definitive view of the ISM in the Local Universe'. F.C. also acknowledges support from the INAF mainstream (1.05.01.86.31). D. R. acknowledges support from the National Science Foundation under grant numbers AST-1614213 and AST-1910107. D. R. also acknowledges support from the Alexander von Humboldt Foundation through a Humboldt Research Fellowship for Experienced Researchers. G. C. J and R. M acknowledge ERC advanced grant 695671 'QUENCH' and support by the Science and Technology Facilities Council (STFC).

\section{References}

Aoyama, S., Hou, K.-C., Hirashita, H., Nagamine, K., \& Shimizu, I. 2018 , MNRAS, 478, 4905

Arnouts, S., Cristiani, S., Moscardini, L., et al. 1999, MNRAS, 310, 540

Beeston, R. A., Wright, A. H., Maddox, S., et al. 2018, MNRAS, 479, 1077 Béthermin, M., Fudamoto, Y., Ginolfi, M., et al. 2020, A\&A, 643, A2

Bianchi, S. 2013, A\&A, 552, A89

Bouwens, R. J., Illingworth, G. D., Oesch, P. A., et al. 2015, ApJ, 803, 34

Calura, F., \& Matteucci, F. 2004, MNRAS, 350, 35

Calura, F., Pipino, A., \& Matteucci, F. 2008, A\&A, 479, 669

Calura, F., Gibson, B. K., Michel-Dansac, L., et al. 2012, MNRAS, 427, 1401

Calura, F., Gilli, R., Vignali, C., et al. 2014, MNRAS, 438, 2765

Calura, F., Pozzi, F., Cresci, G., et al. 2017, MNRAS, 465, 54

Capak, P. L., Carilli, C., Jones, G., et al. 2015, Nature, 522, 455

Cassata, P., Morselli, L., Faisst, A., et al. 2020, A\&A, 643, A6

Chabrier, G. 2003, ApJ, 586, L133

Combes, F., Rex, M., Rawle, T. D., et al. 2012, A\&A, 538, L4

Conley, A., Cooray, A., Vieira, J. D., et al. 2011, ApJ, 732, L35

Cowie, L. L., Songaila, A., Hu, E. M., \& Cohen, J. G. 1996, AJ, 112, 839

da Cunha, E., Groves, B., Walter, F., et al. 2013, ApJ, 766, 13

Dayal, P., \& Ferrara, A. 2018, Phys. Rep., 780, 1

Decarli, R., Walter, F., Gónzalez-López, J., et al. 2019, ApJ, 882, 138

De Masi, C., Vincenzo, F., Matteucci, F., et al. 2019, MNRAS, 483, 2217

Dessauges-Zavadsky, M., Ginolfi, M., Pozzi, F., et al. 2020, A\&A, 643, A5

D'Odorico, V., Feruglio, C., Ferrara, A., et al. 2018, ApJ, 863, L29

Draine, B. T. 2011, Physics of the Interstellar and Intergalactic Medium (Princeton University Press)

Draine, B. T., \& Li, A. 2007, ApJ, 657, 810

Driver, S. P., Andrews, S. K., da Cunha, E., et al. 2018, MNRAS, 475, 2891

Dudzevičiūte, U., Smail, I., Swinbank, A. M., et al. 2021, MNRAS, 500, 942

Dunlop, J. S., McLure, R. J., Biggs, A. D., et al. 2017, MNRAS, 466, 861

Dunne, L., Eales, S. A., \& Edmunds, M. G. 2003, MNRAS, 341, 589

Dunne, L., Gomez, H. L., da Cunha, E., et al. 2011, MNRAS, 417, 1510

Dwek, E. 1998, ApJ, 501, 643

Faisst, A. L., Schaerer, D., Lemaux, B. C., et al. 2020a, ApJS, 247, 61

Faisst, A. L., Fudamoto, Y., Oesch, P. A., et al. 2020b, MNRAS, 498, 4192

Feldmann, R. 2019, Astron. Comput., 29, 100331

Fontana, A., Pozzetti, L., Donnarumma, I., et al. 2004, A\&A, 424, 23

Foreman-Mackey, D., Hogg, D. W., Lang, D., \& Goodman, J. 2013, PASP, 125, 306

Fragkoudi, F., Grand, R. J. J., Pakmor, R., et al. 2020, MNRAS, 494, 5936

Franco, M., Elbaz, D., Béthermin, M., et al. 2018, A\&A, 620, A152 
Fudamoto, Y., Oesch, P. A., Faisst, A., et al. 2020a, A\&A, 643, A4 Fujimoto, S., Silverman, J. D., Bethermin, M., et al. 2020b, ApJ, 900, 1 Gall, C., \& Hjorth, J. 2018, ApJ, 868, 62

Gall, C., Hjorth, J., \& Andersen, A. C. 2011, A\&ARv, 19, 43

Gardner, J. P., Mather, J. C., Clampin, M., et al. 2009, Astrophys. Space Sci. Proc., 10, 1

Giacconi, R., Zirm, A., Wang, J., et al. 2002, ApJS, 139, 369

Gilli, R., Norman, C., Vignali, C., et al. 2014, A\&A, 562, A67

Ginolfi, M., Jones, G. C., Béthermin, M., et al. 2020a, A\&A, 633, A90

Ginolfi, M., Jones, G. C., Béthermin, M., et al. 2020b, A\&A, 643, A7

Gioannini, L., Matteucci, F., \& Calura, F. 2017, MNRAS, 471, 4615

González-López, J., Novak, M., Decarli, R., et al. 2020, ApJ, 897, 91

Gruppioni, C., Pozzi, F., Rodighiero, G., et al. 2013, MNRAS, 432, 23

Gruppioni, C., Béthermin, M., Loiacono, F., et al. 2020, A\&A, 643, A8

Hodge, J. A., \& da Cunha, E. 2020, R. Soc. Open Sci., 7, 200556

Hodge, J. A., Karim, A., Smail, I., et al. 2013, ApJ, 768, 91

Ilbert, O., Arnouts, S., McCracken, H. J., et al. 2006, A\&A, 457, 841

Jones, G. C., Béthermin, M., Fudamoto, Y., et al. 2020, MNRAS, 491, L18

Kaasinen, M., Scoville, N., Walter, F., et al. 2019, ApJ, 880, 15

Kelly, B. C. 2007, ApJ, 665, 1489

Khusanova, Y., Le Fèvre, O., Cassata, P., et al. 2020, A\&A, 634, A97

Khusanova, Y., Béthermin, M., Le Fèvre, O., et al. 2021, A\&A, 649, A152

Koekemoer, A. M., Aussel, H., Calzetti, D., et al. 2007, ApJS, 172, 196

Koekemoer, A. M., Faber, S. M., Ferguson, H. C., et al. 2011, ApJS, 197, 36

Lada, C. J., \& Lada, E. A. 2003, ARA\&A, 41, 57

Laigle, C., McCracken, H. J., Ilbert, O., et al. 2016, ApJS, 224, 24

Laporte, N., Ellis, R. S., Boone, F., et al. 2017, ApJ, 837, L21

Larson, R. B. 1998, MNRAS, 301, 569

Le Fèvre, O., Béthermin, M., Faisst, A., et al. 2020, A\&A, 643, A1

Lemaux, B. C., Le Floc'h, E., Le Fèvre, O., et al. 2014, A\&A, 572, A90

Lemaux, B. C., Le Fèvre, O., Cucciati, O., et al. 2018, A\&A, 615, A77

Leśniewska, A., \& Michałowski, M. J. 2019, A\&A, 624, L13

Li, Q., Narayanan, D., \& Davé, R. 2019, MNRAS, 490, 1425

Liang, L., Feldmann, R., Kereš, D., et al. 2019, MNRAS, 489, 1397

Loiacono, F., Decarli, R., Gruppioni, C., et al. 2021, A\&A, 646, A76

Magnelli, B., Popesso, P., Berta, S., et al. 2013, A\&A, 553, A132

Magnelli, B., Karim, A., Staguhn, J., et al. 2019, ApJ, 877, 45

Magnelli, B., Boogaard, L., Decarli, R., et al. 2020, ApJ, 892, 66

Mancini, M., Schneider, R., Graziani, L., et al. 2015, MNRAS, 451, L70

Matteucci, F. 1994, A\&A, 288, 57

Matteucci, F., \& Francois, P. 1989, MNRAS, 239, 885

McMullin, J. P., Waters, B., Schiebel, D., Young, W., \& Golap, K. 2007, in CASA

Architecture and Applications, eds. R. A. Shaw, F. Hill, \& D. J. Bell, ASP Conf. Ser., 376, 127

Ménard, B., \& Fukugita, M. 2012, ApJ, 754, 116

Narayanan, D., Davé, R., Johnson, B. D., et al. 2018, MNRAS, 474, 1718

Negrello, M., Amber, S., Amvrosiadis, A., et al. 2017, MNRAS, 465, 3558
Ono, Y., Ouchi, M., Harikane, Y., et al. 2018, PASJ, 70, S10

Orellana, G., Nagar, N. M., Elbaz, D., et al. 2017, A\&A, 602, A68

Palla, M., Calura, F., Matteucci, F., et al. 2020, MNRAS, 494, 2355

Pavesi, R., Riechers, D. A., Faisst, A. L., Stacey, G. J., \& Capak, P. L. 2019, ApJ, 882,168

Péroux, C., \& Howk, J. C. 2020, ARA\&A, 58, 363

Pipino, A., Fan, X. L., Matteucci, F., et al. 2011, A\&A, 525, A61

Planck Collaboration XXV. 2011, A\&A, 536, A25

Popping, G., Somerville, R. S., \& Galametz, M. 2017, MNRAS, 471, 3152

Pozzi, F., Calura, F., Gruppioni, C., et al. 2015, ApJ, 803, 35

Pozzi, F., Calura, F., Zamorani, G., et al. 2020, MNRAS, 491, 5073

Privon, G. C., Narayanan, D., \& Davé, R. 2018, ApJ, 867, 102

Riechers, D. A., Bradford, C. M., Clements, D. L., et al. 2013, Nature, 496 , 329

Riechers, D. A., Carilli, C. L., Capak, P. L., et al. 2014, ApJ, 796, 84

Roca-Fàbrega, S., Valenzuela, O., Colín, P., et al. 2016, ApJ, 824, 94

Rodighiero, G., Daddi, E., Baronchelli, I., et al. 2011, ApJ, 739, L40

Roelfsema, P. R., Shibai, H., Armus, L., et al. 2018, PASA, 35, e030

Romano, M., Cassata, P., Morselli, L., et al. 2020, MNRAS, 496, 875

Rowan-Robinson, M., Oliver, S., Wang, L., et al. 2016, MNRAS, 461, 1100

Salpeter, E. E. 1955, Vistas Astron., 1, 283

Scalo, J. M. 1986, Fund. Cosmic Phys., 11, 1

Schaerer, D., Ginolfi, M., Bethermin, M., et al. 2020, A\&A, 643, A3

Schmidt, M. 1959, ApJ, 129, 243

Schurer, A., Calura, F., Silva, L., et al. 2009, MNRAS, 394, 2001

Scoville, N., Aussel, H., Brusa, M., et al. 2007, ApJS, 172, 1

Scoville, N., Aussel, H., Sheth, K., et al. 2014, ApJ, 783, 84

Scoville, N., Sheth, K., Aussel, H., et al. 2016, ApJ, 820, 83

Scoville, N., Lee, N., Vanden Bout, P., et al. 2017, ApJ, 837, 150

Shull, J. M., Smith, B. D., \& Danforth, C. W. 2012, ApJ, 759, 23

Simpson, J. M., Smail, I., Swinbank, A. M., et al. 2017, ApJ, 839, 58

Sommovigo, L., Ferrara, A., Pallottini, A., et al. 2020, MNRAS, 497, 956

Speagle, J. S., Steinhardt, C. L., Capak, P. L., \& Silverman, J. D. 2014, ApJS, 214,15

Spitoni, E., Silva Aguirre, V., Matteucci, F., Calura, F., \& Grisoni, V. 2019, A\&A, 623, A60

Spitoni, E., Calura, F., Mignoli, M., et al. 2020, A\&A, 642, A113

Thomas, R., Le Fèvre, O., Scodeggio, M., et al. 2017, A\&A, 602, A35

Valiante, R., Schneider, R., Salvadori, S., \& Bianchi, S. 2011, MNRAS, 416, 1916

Vijayan, A. P., Clay, S. J., Thomas, P. A., et al. 2019, MNRAS, 489, 4072

Vlahakis, C., Dunne, L., \& Eales, S. 2005, MNRAS, 364, 1253

Walter, F., Decarli, R., Aravena, M., et al. 2016, ApJ, 833, 67

Watson, D., Christensen, L., Knudsen, K. K., et al. 2015, Nature, 519, 327

Weidner, C., \& Kroupa, P. 2005, ApJ, 625, 754

Yan, L., Sajina, A., Loiacono, F., et al. 2020, ApJ, 905, 147

Zanella, A., Daddi, E., Magdis, G., et al. 2018, MNRAS, 481, 1976 


\section{Appendix A: Dust mass estimates}

Table A.1. $\mathrm{M}_{\text {dust }}$ estimates for the 23 ALPINE continuum detected sources.

\begin{tabular}{lc}
\hline \hline Target Name & $\begin{array}{c}\log \mathrm{M}_{\text {dust }} \\
{\left[\log _{10}\left(\mathrm{M} / \mathrm{M}_{\odot}\right)\right]}\end{array}$ \\
\hline CANDELS_GOODSS_19 & $8.21 \pm 0.19$ \\
CANDELS_GOODSS_32 & $8.12 \pm 0.21$ \\
DEIMOS_COSMOS_396844 & $8.31 \pm 0.19$ \\
DEIMOS_COSMOS_417567 & $8.22 \pm 0.21$ \\
DEIMOS_COSMOS_422677 & $8.33 \pm 0.22$ \\
DEIMOS_COSMOS_460378 & $7.95 \pm 0.21$ \\
DEIMOS_COSMOS_488399 & $8.32 \pm 0.18$ \\
DEIMOS_COSMOS_493583 & $8.14 \pm 0.22$ \\
DEIMOS_COSMOS_494057 & $8.16 \pm 0.18$ \\
DEIMOS_COSMOS_539609 & $8.13 \pm 0.21$ \\
DEIMOS_COSMOS_552206 & $8.35 \pm 0.20$ \\
DEIMOS_COSMOS_683613 & $8.29 \pm 0.19$ \\
DEIMOS_COSMOS_818760 & $8.81 \pm 0.17$ \\
DEIMOS_COSMOS_848185 & $8.38 \pm 0.18$ \\
DEIMOS_COSMOS_873756 & $8.91 \pm 0.17$ \\
DEIMOS_COSMOS_881725 & $8.32 \pm 0.25^{(a)}$ \\
vuds_cosmos_5100822662 & $8.09 \pm 0.18$ \\
vuds_cosmos_5100969402 & $8.29 \pm 0.21$ \\
vuds_cosmos_5100994794 & $7.85 \pm 0.21$ \\
vuds_cosmos_5101209780 & $8.27 \pm 0.23^{(a)}$ \\
vuds_cosmos_5101218326 & $8.44 \pm 0.18$ \\
vuds_cosmos_5180966608 & $8.39 \pm 0.19$ \\
vuds_efdcs_530029038 & $7.85 \pm 0.26^{(a)}$ \\
\hline
\end{tabular}

Notes. The dust masses were estimated using a modified black-body (MBB) spectrum, under the approximation of thin emission, assuming $\mathrm{T}_{\text {dust }}=25 \mathrm{~K}$ and the spectral index $\beta=1.8$ (see Sect. 3). ${ }^{(a)}$ Multi-component objects (see Appendix D.2 and Table D.1 in Béthermin et al. 2020). For DEIMOS_COSMOS_881725 the sum of the components was considered; for vuds_cosmos_51012097 and vuds_efdcs_530029038 only the fluxes of the central targets were considered since the companions are likely separated objects (see Sect. 2). 